\title{
ANFOTERICINA B: UMA REVISÃO SOBRE SUAS DIFERENTES FORMULAÇÕES, EFEITOS ADVERSOS E TOXICIDADE
}

\author{
AMPHOTERICIN B: A REVIEW ON DIFFERENT FORMULATIONS, SIDE \\ EFFECTS, AND TOXICITY
}

Diego Rodrigues Falci', Alessandro Comarú Pasqualotto ${ }^{2,3}$

\section{RESUMO}

A incidência de infecções fúngicas invasivas tem aumentado, como consequência do contingente cada vez maior de pacientes com imunossupressão. O tratamento de infecções fúngicas com anfotericina $B(A m B)$ está associado a efeitos adversos importantes, como nefrotoxicidade e toxicidade hematológica. Nesta revisão buscou-se abordar os estudos sobre AmB nas diferentes formulações, focando em suas características farmacológicas e toxicidade. Formulações lipídicas de AmB estão associadas a um risco menor de nefrotoxicidade, entretanto ainda há controvérsia sobre diferenças entre as duas formulações lipídicas de AmB disponíveis. Diferenças em relação ao perfil imunomodulatório e ligação a lipoproteínas podem explicar parte das diferenças clínicas existentes entre as formulações de AmB. A maioria dos estudos clínicos que avaliou a nefrotoxicidade associada à AmB em diferentes formulações não utilizou critérios validados para classificação do dano renal, o que dificulta sua comparação. A toxicidade hematológica relacionada ao uso de AmB é um fenômeno descrito desde os primórdios do seu uso clínico, entretanto poucos dados existem sobre sua frequência, fatores de risco e impacto nos desfechos clínicos. Dados precisos, e adequados ao contexto local, sobre a toxicidade de AmB nas suas diferentes formulações são necessários para uma adequada avaliação dos aspectos de farmacoeconomia e custo-efetividade.

Palavras-chave: Anfotericina B; micoses; toxicidade; anemia; lesão renal aguda

\section{ABSTRACT}

Invasive fungal infections have emerged in recent years, as a consequence of increasing numbers of immunosuppressed patients. Treatment of these conditions with amphotericin $\mathrm{B}(\mathrm{AmB})$ has been associated with important side effects, such as nephrotoxicity and hematological toxicity. In this review we aimed to assess studies about different formulations of AmB, focusing on pharmacological properties and toxicity. Lipid formulations of AmB have been linked to a lower risk of nephrotoxicity; however, there is still controversy about differences between the two available lipid formulations. Differences in immunomodulatory profile and lipoprotein binding could partly explain clinical inequalities among AmB formulations. Most clinical trials that evaluated AmB-associated nephrotoxicity did not use validated criteria for renal injury classification, impairing comparability. Hematological toxicity associated with AmB treatments is an occurrence described since the beginning of its clinical use; nevertheless, few data exist about its frequency, risk factors, and clinical impact. Clear and more precise information, derived from local studies, is needed to an adequate evaluation about pharmacoeconomic aspects of AmB treatment and cost-effectiveness of lipid formulations.

Keywords: Amphotericin B; mycoses; toxicity; anemia; acute kidney injury
Clin Biomed Res. 2015;35(2):65-82

1 Serviço de Infectologia, Hospital de Clínicas. Porto Alegre, RS, Brasil.

2 Departamento de Clínica Médica, Universidade Federal de Ciências da Saúde de Porto Alegre (UFCSPA). Porto Alegre, RS, Brasil.

3 Laboratório de Biologia Molecular, Santa Casa de Misericórdia. Porto Alegre, RS, Brasil.

Autor correspondente:

Diego Rodrigues Falci

E-mail: dfalci@hcpa.edu.br

Serviço de Infectologia, Hospital de

Clínicas

Rua Ramiro Barcelos, 2350.

90035-903, Porto Alegre, RS, Brasil. 
As infecções fúngicas sistêmicas representam um problema emergente na prática clínica atual. Sua incidência tem aumentado, principalmente com o uso intensivo de cateteres intravasculares, antibióticos de largo espectro e com a frequência cada vez maior de condições associadas à imunossupressão, tais como transplantes, neoplasias com tratamento quimioterápico e síndrome da imunodeficiência adquirida (Aids). As infecções fúngicas sistêmicas associam-se à elevada morbimortalidade, a despeito de terapia antifúngica adequada ${ }^{1,2}$. O difícil diagnóstico e a mortalidade elevada associados às micoses sistêmicas acarretam, na maioria das vezes, necessidade de tratamentos empíricos ou até mesmo profiláticos. A emergência de fungos incomuns ou resistentes aos agentes antifúngicos pode complicar ainda mais esse cenário ${ }^{3,4}$.

Um levantamento recente, que envolveu a participação de 183 hospitais nos Estados Unidos, avaliando a epidemiologia das infecções hospitalares, demonstrou a importância da infecção fúngica nesse contexto: Candida spp. foi a etiologia mais frequente das infecções primárias de corrente sanguínea, com uma estimativa de 15,000 casos/ano naquele país ${ }^{5}$, demonstrando a importância crescente da doença fúngica no âmbito da infecção hospitalar.

Felizmente, as opções para tratamento sistêmico das infecções fúngicas têm sido ampliadas de modo considerável nos últimos anos. A anfotericina $\mathrm{B}$ deoxicolato (d-AmB), considerada por muitos anos o padrão-ouro para tratamento dessas condições, tem sido progressivamente substituída por alternativas mais modernas e associadas a maior eficácia e/ou menor toxicidade, incluindo as formulações lipídicas de anfotericina $B$ - anfotericina lipossomal (L-AmB), anfotericina em dispersão coloidal (ABCD) e anfotericina em complexo lipídico (ABLC), triazólicos de segunda geração (voriconazol e posaconazol), e equinocandinas (caspofungina, anidulafungina $e$ micafungina). A despeito da disponibilidade de novas drogas antifúgincas, d-AmB ainda exerce um papel importante na prática clínica ${ }^{6-8}$, particularmente em ambientes onde os recursos financeiros são limitados, como na maioria dos hospitais públicos brasileiros.

O principal efeito adverso associado ao uso de d-AmB é a nefrotoxicidade. Além da nefrotoxicidade, outros efeitos adversos, como reações agudas infusionais, anemia, neutropenia, plaquetopenia e alterações de enzimas hepáticas, estão descritos ${ }^{7,9-15}$. Os efeitos adversos da d-AmB, especialmente a nefrotoxicidade, limitam seu uso de forma importante, pois trazem consequências significativas do ponto de vista de desfechos clínicos e custos hospitalares ${ }^{16,17}$. As duas formulações lipídicas de anfotericina $B$ disponíveis no Brasil (L-AmB e ABLC) proporcionam uma inequívoca redução na frequência de nefrotoxicidade, quando comparadas à d-AmB ${ }^{18,19}$. Entretanto, não está totalmente clara na literatura a existência de vantagem de uma formulação lipídica em relação à outra ${ }^{20}$. Ainda está por ser esclarecida, também, eventual vantagem de uma formulação sobre a outra em determinadas populações e situações clínicas específicas. Ademais, a real frequência de nefrotoxicidade por d-AmB não foi ainda demonstrada, em nosso país, com base em estudos prospectivos contemporâneos. Já na pediatria, não há consenso em relação à superioridade das formulações lipídicas, e em geral estas estão sendo reservadas para pacientes não neonatos com fatores de risco ou nefropatia prévia; em neonatos, a indicação de formulação lipídica de anfotericina B é ainda muito limitada ${ }^{21}$.

\section{ANFOTERICINA B}

$A$ anfotericina $B(A m B)$ é um antimicrobiano da classe dos polienos, isolado pela primeira vez em 1955 a partir de culturas de Streptomyces nodosus obtidas no delta do rio Orinoco, na Venezuela ${ }^{6,7}$. A baixa solubilidade é uma característica dos antimicrobianos polienos.

A anfotericina é uma molécula anfipática (figura 1), ou seja, possui uma região hidrofílica e uma região hidrofóbica, esta última a torna insolúvel em meios aquosos. A solubilidade aquosa é adquirida através da formulação com o deoxicolato de sódio ou uma variedade de carreadores lipídicos ${ }^{22}$. A AmB é, via de regra, administrada por via intravenosa, misturada com um agente solubilizante como o deoxicolato de sódio. Essa combinação de AmB com deoxicolato de sódio é a formulação mais utilizada na prática clínica para o tratamento de infecções fúngicas, desde 1958, especialmente em países em desenvolvimento ${ }^{6,7}$. AmB tem um amplo espectro de ação e eficácia demonstrada em candidíase, criptococose, aspergilose, histoplasmose, blastomicose, coccidiomicose, mucormicose, esporotricose, fusariose e outras feohifomicoses. Alguns

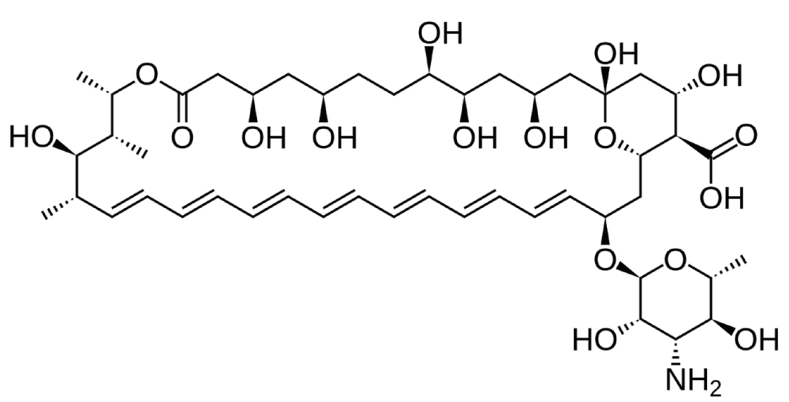

Figura 1: Estrutura molecular da anfotericina B. 
fungos raros, como Trichosporon spp., Aspergillus terreus, Scedosporium spp. e Malassezia furfur são intrinsecamente resistentes a $\mathrm{AmB}^{6}$, mas ela ainda é o pilar fundamental na terapia da criptococose disseminada, a doença fúngica com maior impacto em mortalidade no mundo, causando mais de 600,000 mortes por ano ${ }^{8}$. Além de ser indispensável para o tratamento das infecções fúngicas, é também um antimicrobiano importante para o tratamento da leishmaniose visceral e cutânea ${ }^{7}$.

O mecanismo de ação da AmB ainda não está completamente elucidado, mas pode ser dividido entre efeitos diretos sobre a membrana celular e efeitos intracelulares de indução de estresse oxidativo ${ }^{22}$. O efeito na membrana baseia-se na sua interação com o ergosterol, um esteroide essencial da membrana celular dos organismos fúngicos, e de alguns protozoários (gênero Leishmania e amebas) ${ }^{7}$. A estrutura poliênica da molécula forma complexos com o ergosterol, causando a formação de poros na membrana que resultam em perda de sua integridade e rápido extravasamento de potássio e outros íons, ocasionando a morte celular ${ }^{7}$. O sequestro do ergosterol pelas moléculas de AmB também resulta em efeito tóxico para as células do patógeno, visto que o ergosterol é essencial para múltiplos processos celulares, como endocitose, fusão de vacúolos e estabilização de proteínas da membrana ${ }^{22}$. A indução do estresse oxidativo é outro mecanismo de ação importante da AmB e também responsável pelo seu efeito antimicrobiano. Evidências iniciais já mostravam que o efeito sobre a membrana não parecia ser o único mecanismo pelo qual a molécula exercia suas propriedades farmacológicas. Tem sido cada vez mais demonstrado que a AmB induz o estresse oxidativo celular e o aumento na formação de radicais livres, evidenciado também pela expressão dos genes de estresse celular, por mecanismos ainda não completamente conhecidos ${ }^{22}$.

As anfotericinas apresentam também potente efeito imunomodulador, porém distinto entre as diferentes formulações. Ad-AmB é um medicamento com ação pró-inflamatória ${ }^{23}$. Já as formulações lipídicas apresentam um efeito distinto e serão abordadas posteriormente. A d-AmB estimula a produção de citocinas inflamatórias através do toll-like receptor (TLR)-2, promovendo a resposta imunitária do tipo Thelper-1 (Th1). Isto leva a ativação de macrófagos e produção de superóxidos e óxido nítrico, melhorando a resposta do hospedeiro à infecção fúngica - entretanto, em um hospedeiro não imunossuprimido, isso pode levar a uma exacerbação da resposta e piora no quadro clínico. Ainda, essa cascata inflamatória também é responsável pelas reações agudas infusionais, comumente vistas após a administração de d-AmB ${ }^{22,23}$. Outro efeito adverso importante da AmB é a nefrotoxicidade. O mecanismo pelo qual a AmB causa nefrotoxicidade é mediado principalmente em nível celular pela apoptose ${ }^{24}$, e será aprofundado mais adiante nesta revisão.

\section{FORMULAÇÕES LIPÍDICAS DE ANFOTERICINA B}

Com o objetivo de reduzir principalmente a nefrotoxicidade, buscou-se o desenvolvimento de formulações farmacêuticas inovadoras de AmB. Para alcançar somente os tecidos-alvo com máxima concentração e seletividade, a concentração sérica da droga livre deve ser mantida baixa. O interesse tecnológico está focado no desenvolvimento de sistemas "carreadores", coloidais ou através de partículas como lipossomos ou nanopartículas. Essas novas formulações de AmB combinadas a esses sistemas carreadores são coletivamente denominadas formulações lipídicas de anfotericina ${ }^{7}$.

Existem, hoje, três formulações lipídicas disponíveis no mercado: Ambisome ${ }^{\circledR}$ é a anfotericina B lipossomal (L-AmB); Abelcet $^{\circledR}$ é o complexo lipídico de anfotericina B (ABLC); Amphotec ${ }^{\circledR}$ ou Amphocil $^{\circledR}$ é a dispersão coloidal de anfotericina $B(A B C D)$. As duas primeiras (L-AmB e ABLC) são as mais utilizadas na prática clínica e disponíveis no mercado brasileiro, e serão abordadas nesta revisão.

\section{ANFOTERICINA B LIPOSSOMAL}

L-AmB é o nome da formulação na qual a anfotericina B está integrada a lipossomos unilamelares. Em conjunto com a molécula de AmB, a L-AmB é composta por fosfatidilcolina de soja hidrogenada, distearoil fosfatidilglicerol e colesterol. Os componentes são fornecidos liofilizados como um pó e devem ser reconstituídos em água imediatamente antes do uso, produzindo lipossomos com um diâmetro médio de 60-70 nm. A estabilidade dos lipossomos depende fundamentalmente da natureza dos fosfolipídios utilizados para a sua formação. Os componentes utilizados para a formação de L-AmB (fosfatidilcolina de soja hidrogenada, distearoil fosfatidilglicerol) têm elevada temperatura de transição e foram utilizados por terem estabilidade a $37^{\circ} \mathrm{C}$. O colesterol foi adicionado para proporcionar mais estabilidade na formulação e para manter a AmB dentro da bicamada do lipossomo, já que a AmB liga-se ao colesterol ${ }^{25}$. Quando os lipossomos contendo AmB entram em contato com células fúngicas, a matriz lipossômica é degradada, e a AmB é liberada para ligar-se preferencialmente ao ergosterol na membrana celular fúngica, levando a sua desintegração ${ }^{7}$. 
A nefrotoxicidade reduzida apresentada por L-AmB pode estar relacionada a diversos mecanismos. L-AmB, como outras formulações lipídicas, tem afinidade maior por lipoproteínas de alta densidade (HDL) do que lipoproteínas de baixa densidade $(L D L)^{26}$. A ligação às moléculas de HDL promove a captação pelo sistema reticuloendotelial, que tem elevada expressão de receptores HDL (diferente do tecido renal, com expressão aumentada de receptores $L D L$ ). A captação preferencial de L-AmB pelos macrófagos hepáticos e esplênicos do sistema reticuloendotelial e concentração renal baixa contribui para a redução da nefrotoxicidade e para a melhora do índice terapêutico de L-AmB, em comparação à $\mathrm{d}-\mathrm{AmB}^{26,27}$. Um outro potencial mecanismo para a redução de nefrotoxicidade é a difusão seletiva da AmB para as células fúngicas versus as células do hospedeiro ${ }^{26,27}$. A liberação da AmB do veículo lipídico carreador, mediada pelas fosfolipases fúngicas na membrana celular fúngica, aumenta ainda mais a especificidade hospedeiro-patógeno das formulações lipídicas de $\mathrm{AmB}^{26}$.

As reações agudas infusionais ocorrem em menor frequência do que com d-AmB e não têm seu mecanismo fisiopatológico completamente elucidado. Diferentemente de d-AmB, que apresenta reações agudas infusionais mediadas por fator de necrose tumoral alfa (TNF- $\alpha$ ) e interleucina-6 (IL-6), a L-AmB tem uma síndrome distinta, idiossincrásica, aparentemente mediada por histamina e possivelmente a fração C5a do complemento ${ }^{26}$.

Estudos pré-clínicos e clínicos (fase I e II) indicaram que L-AmB foi bem tolerada e apresenta nefrotoxicidade reduzida em comparação à d-AmB ${ }^{28-31}$. Outras condições clínicas foram avaliadas, nos estudos iniciais com esta formulação, com eficácia comparável à $\mathrm{d}-\mathrm{AmB}^{32-36}$. A L-AmB despontou como uma alternativa com um índice terapêutico muito mais favorável do que d-AmB, o que a tornou particularmente promissora para o tratamento de condições graves, como a aspergilose invasiva, histoplasmose disseminada e também a leishmaniose visceral, em que concentrações maiores de anfotericina $B$ são necessárias para o controle infeccioso ${ }^{37-40}$.

Neste contexto, Walsh et al. conduziram um ensaio clínico randomizado, multicêntrico, duplo-cego, comparando L-AmB e d-AmB, no tratamento empírico de infecções fúngicas invasivas, em pacientes com neutropenia e febre persistente. Foram estudados 702 pacientes; destes, 343 receberam L-AmB e 344 receberam d-AmB. Os desfechos clínicos de sucesso do tratamento foram similares entre os dois grupos (50 e 49\%, respectivamente). Em relação à mortalidade, resolução da febre e descontinuação da droga em razão de efeitos adversos ou falta de eficácia, os resultados também foram similares nos dois grupos. Houve menos infecções fúngicas de escape (infecção na vigência do antifúngico) entre os pacientes que receberam L-AmB $(3,2 \%)$ do que nos pacientes que receberam a d-AmB $(7,9 \%)$. Houve diferença significativa quando analisadas as reações infusionais agudas, com o grupo que recebeu L-AmB apresentando menor incidência. Quanto à frequência de nefrotoxicidade, definida como um aumento da creatinina sérica de duas vezes o valor da normalidade, também se observou diferença significativa: apresentaram nefrotoxicidade $19 \%$ dos pacientes tratados com L-AmB, em comparação com $34 \%$ dos pacientes tratados com d-AmB $(p<0,001)^{18}$. Os pacientes que receberam L-AmB apresentaram melhor sustentabilidade da função glomerular e tubular do que aqueles que receberam d-AmB, como evidenciado pelos índices menores de azotemia e hipocalemia.

Esse importante ensaio clínico randomizado demonstrou similaridade entre os desfechos clínicos dos dois grupos de tratamento, L-AmB e d-AmB. Entretanto, a L-AmB foi mais efetiva em reduzir a frequência de infecções fúngicas de escape, reações infusionais agudas e nefrotoxicidade. $\mathrm{O}$ estudo estabeleceu claramente que a L-AmB apresenta menor toxicidade do que a d-AmB; o poder estatístico dessas observações é embasado pelo monitoramento, prospectivo e duplo-cego, à beira do leito, de mais de 7 mil infusões. Os benefícios de redução das reações agudas infusionais podem ser particularmente importantes para pacientes criticamente enfermos, que têm uma tolerância menor aos eventos cardiorrespiratórios que podem ocorrer nessas reações ${ }^{18,41}$.

A imunomodulação característica das anfotericinas ocorre de forma diferente nas formulações lipídicas e particularmente com L-AmB, tendo esta um efeito anti-inflamatório. L-AmB provoca down-regulation na expressão gênica de citocinas inflamatórias nos macrófagos, além de interagir com os receptores TLR-4 nos neutrófilos (diferentemente de d-AmB, que se liga primariamente aos TLR-2 com efeito pró-inflamatório ${ }^{22,23}$. Com essa mudança na sinalização de TLR-2 para TLR-4, L-AmB potencialmente estaria associada com um menor grau de inflamação durante a infecção fúngica, uma propriedade importante nos pacientes expostos a corticosteroides. A diferença no padrão de produção de citocinas pode explicar a chance menor de reações agudas infusionais com L-AmB. O efeito anti-inflamatório dessa formulação deve-se, pelo menos parcialmente, ao carreador (lipossomo) ${ }^{23}$. 


\section{ANFOTERICINA B COMPLEXO LIPÍDICO}

Anfotericina B complexo lipídico (ABLC) é composta por estruturas tipo fita, com um tamanho entre 1600 e $11000 \mu \mathrm{m}$, em uma razão molar 1:1 de AmB e fosfolipídios (uma mistura de L-a-dimiristoilfosfatidilcolina e L-a-dimiristoilfosfatidilglicerol na razão de $7: 3)^{42,43}$. ABLC também foi desenvolvida com o intuito de reduzir a toxicidade e maximizar a utilidade da AmB para o tratamento de infecções.

A presença de fosfolipídios com carga iônica negativa (dimistoril fosfaditilglicerol) e carga iônica neutra (dimistoril fosfaditilcolina) ocasiona a redução da transferência dos lipídios (através da proteína transferidora de lipídios) de HDL para LDL, e é responsável pela distribuição maior de $A B L C$ na fração HDL. Como resultado, acontece uma interação menor de ABLC com as células tubulares renais, por conta da escassez de receptores HDL naquele sítio, fenômeno que ocorre também com L-AmB ${ }^{44}$. Isso explica a seletividade maior dessas preparações. Fosfolipases liberadas pelo tecido muscular liso dos vasos, em conjunto com fosfolipases de macrófagos e aquelas secretadas pelas células fúngicas infectantes, quebram os lipídios do complexo e liberam a AmB diretamente no foco da infecção ${ }^{45}$.

O efeito imunomodulador das formulações de AmB pode ser compreendido através da sua interação com os TLR. As formulações lipídicas, como já mencionado, estimulam predominantemente resposta TLR-4 - com efeito anti-inflamatório - diferentemente de d-AmB, que interage com TLR-2, desencadeando uma potente resposta pró-inflamatória com liberação de citocinas inflamatórias e ativação de macrófagos ${ }^{22,23}$. Esses elementos estão na gênese das reações agudas infusionais observadas com as preparações de AmB. Apesar de ABLC in vitro confirmar essa resposta TLR-4, com down-regulation ou com ausência de efeito sobre a expressão gênica de citocinas inflamatórias em macrófagos, a experiência clínica não demonstrou uma redução na incidência de reações agudas infusionais com essa formulação especificamente ${ }^{44}$.

Em uma avaliação clínica inicial de ABLC, logo após a sua aprovação pela agência de controle de medicamentos norte-americana (FDA) para casos de intolerância ou refratariedade à terapia com d-AmB, Walsh e colaboradores avaliaram 556 pacientes que receberam $A B L C$ para infecção fúngica invasiva presumida ou comprovada. Todos os pacientes apresentavam algum dos seguintes critérios para inclusão no estudo: falha em um tratamento antifúngico prévio, incluindo d-AmB administrada em uma dose cumulativa de pelo menos 500 mg; ocorrência de nefrotoxicidade em tratamento antifúngico prévio; toxicidade aguda grave; nefropatia prévia, contra-indicando o tratamento com d-AmB. A dose utilizada no estudo foi de $5 \mathrm{mg} / \mathrm{kg} / \mathrm{dia}$, mas podendo ser reduzida a critério dos investigadores. $\mathrm{O}$ achado interessante deste estudo é que a função renal dos pacientes avaliados melhorou durante o curso da terapia, alcançando significância estatística após a terceira semana. Essa melhora na função renal ocorreu principalmente naqueles pacientes incluídos no estudo por apresentarem nefropatia prévia ou intolerância a tratamento anterior com d-AmB. A taxa de resposta global (parcial ou completa) foi de $57 \%$ no estudo, variando bastante entre as diferentes infecções fúngicas observadas ${ }^{19}$. Embora o estudo tenha apresentado limitações pelo seu delineamento, aberto e sem comparador, o mérito esteve em demonstrar um perfil de segurança superior ao de d-AmB (em relação a nefrotoxicidade) em uma série grande de pacientes.

Outro trabalho demonstrou nefrotoxicidade reduzida de $\mathrm{ABLC}$, comparando à d-AmB em pacientes com candidemia. Em um estudo multicêntrico, 194 pacientes (129 com ABLC e 65 com d-AmB) foram arrolados. A dose utilizada de ABLC foi de $5 \mathrm{mg} / \mathrm{kg} / \mathrm{dia}$. Eficácia e eventos adversos não renais foram equivalentes entre os grupos; $A B L C$ foi significativamente menos nefrotóxico que d-AmB $(49 \% \text { versus } 28 \% ; p=0,006)^{46}$.

A eficácia e segurança de ABLC em pacientes submetidos a transplante de células-tronco hematopoiéticas foram avaliadas em um estudo aberto, não comparativo, que envolveu 95 pacientes $^{45}$. Esse estudo confirmou os trabalhos anteriores ${ }^{19,46}$ em relação a um perfil favorável de $A B L C$ no que diz respeito à nefrotoxicidade. Apenas dois pacientes descontinuaram o medicamento por conta de nefrotoxicidade; entretanto, $13 \%$ dos pacientes apresentaram uma elevação de $100 \%$ na creatinina sérica. De forma semelhante a um trabalho anterior ${ }^{19}$, também foi observada uma melhora na função renal nos pacientes que iniciaram o estudo com creatinina alterada, da segunda semana de tratamento em diante ${ }^{45}$.

A comparação entre ABLC e d-AmB no tratamento da meningite criptocócica associada à Aids foi estudada em um trabalho que envolveu 55 pacientes: 38 receberam $A B L C$ e 17, d-AmB. Os pacientes que receberam $A B L C$ foram divididos em três coortes, conforme a dose utilizada: $1,2 \mathrm{mg} / \mathrm{kg} / \mathrm{dia}, 2,5 \mathrm{mg} / \mathrm{kg} / \mathrm{dia}$ e $5 \mathrm{mg} / \mathrm{kg} / \mathrm{dia}$. Foram avaliados aspectos de eficácia clínica e segurança (nefrotoxicidade e toxicidade hematológica). Embora o estudo tenha apresentado limitações, principalmente por diferenças pré-tratamento, entre os grupos, pode-se concluir que ABLC foi igualmente eficaz e melhor tolerada que d-AmB, sendo a incidência de nefrotoxicidade menor com 
$A B L C$, conforme a dose utilizada ${ }^{47}$. A influência da dose de ABLC foi enfatizada em um outro trabalho, que avaliou a utilização de $1 \mathrm{mg} / \mathrm{kg} / \mathrm{dia}$, e encontrou apenas $8 \%$ de nefrotoxicidade, comparado a $32 \%$ com d-AmB ${ }^{48}$. Ressalta-se, no entanto, que este estudo, além de envolver um número pequeno de pacientes, arrolou pacientes com neutropenia febril e sem infecção fúngica comprovada, o que pode levantar questionamentos sobre a eficácia desse regime de dosagem. Por outro lado, em um trabalho de farmacovigilância, que incluiu 93 pacientes, não foi encontrada qualquer correlação entre a dose utilizada (seja a dose diária prescrita ou a dose cumulativa recebida pelos pacientes) e a incidência de nefrotoxicidade ${ }^{49}$.

Em uma revisão sistemática dos estudos que avaliaram $A B L C$, conclui-se que este agente, na dose de $5 \mathrm{mg} / \mathrm{kg} / \mathrm{dia}$, é tão eficaz quanto d-AmB e menos tóxico. Uma incidência elevada de reações agudas infusionais é mencionada, mas diminuída em importância em razão de seu fácil manejo e baixa morbidade ${ }^{50}$. Embora esteja bem documentado que L-AmB tenha uma incidência claramente menor de reações agudas infusionais do que $\mathrm{d}-\mathrm{AmB}$, o mesmo não foi demonstrado de forma inequívoca com $A B L C$, que parece estar no mesmo patamar do que d-AmB, em relação a esse aspecto ${ }^{46,51}$.

\section{FARMACOCINÉTICA E FARMACODINÂMICA}

A absorção gastrintestinal de AmB e, consequentemente, sua biodisponibilidade são mínimas. Alguns dados detalhados do metabolismo da AmB ainda não são totalmente conhecidos. Mesmo após 40 anos de uso clínico, o regime posológico de d-AmB ainda não está completamente definido, sendo indicado pelo fabricante a infusão diária de 2 a 6 horas. Quando a d-AmB é administrada e entra na corrente sanguínea, o deoxicolato separa-se prontamente da AmB. As moléculas de AmB, então, ligam-se às lipoproteínas plasmáticas (acima de $95 \%)^{52}$, divididas entre HDL e LDL. Inicialmente, uma proporção liga-se à HDL, com uma subsequente compensação para a LDL através da ação da proteína de transferência de colesterol esterificado ou proteína de transferência lipídica ${ }^{44}$.

A maior parte do fármaco deixa a circulação sistêmica e é deslocada para o fígado e outros órgãos. As concentrações de AmB em áreas inflamadas, tais como peritônio, pleura e articulações são aproximadamente dois terços do nível sérico. AAmB penetra pouco nas meninges em seu estado normal ou com inflamação, como também no cérebro, saliva, secreções brônquicas, humor vítreo, líquido amniótico, músculos e ossos ${ }^{53}$. Aproximadamente $20-30 \%$ da
AmB é metabolizada no fígado e excretada com a bile nas fezes. Cerca de $2-5 \%$ da AmB encontrada na urina não foi metabolizada e permanece biologicamente ativa ${ }^{7}$. Bekersky e colaboradores (2002) constataram que até dois terços de d-AmB são excretados inalterados na urina $(20,6 \%)$ e fezes $(42,5 \%)$, sugerindo que não há uma metabolização extensa do medicamento ${ }^{54}$.

Os estudos existentes classificam os polienos -AmB - como compostos concentração-dependentes ${ }^{55}$. Entretanto há resultados conflitantes nesses estudos, como por exemplo a observação de ação antifúngica similar às primeiras horas e persistente após 24 horas de administração, o que pode refletir in vivo a difusão lenta nos tecidos desses compostos. Embora esteja estabelecido que a AmB claramente tem atividade concentração-dependente ${ }^{55}$, existe provavelmente um efeito de teto da fração livre (bioativa) da droga, baseado na ligação às proteínas plasmáticas e solubilidade, o que poderia variar de acordo com o sítio da infecção em órgãos como os rins, pulmões, fígado e cérebro ${ }^{52}$.

O objetivo farmacocinético de qualquer tratamento antifúngico é atingir concentrações terapêuticas no sítio da infecção. Desta forma, devemos considerar, além do medicamento, qual o fungo que está causando a infecção e aonde está localizada ou compartimentalizada. A maioria dos fungos patogênicos encontra-se no meio extracelular; logo a concentração sérica seria um marcador confiável para terapêutica adequada. Entretanto, em infecções compartimentalizadas como as do sistema nervoso central, tais como a meningoencefalite criptocócica, a concentração no parênquima cerebral pode ser mais importante. Estudos em animais demonstraram a penetração equiparável no parênquima cerebral de $\mathrm{d}$-AmB em relação às outras formulações de $A m B^{55,56}$. De fato, os tratamentos com d-AmB (1 mg/kg/dia) e L-AmB ( $5 \mathrm{mg} / \mathrm{kg} / \mathrm{dia}$ ) proporcionaram as maiores concentrações de pico plasmático (CMax) e área sob a curva (AUC), em comparação as outras formulações de AmB (ABCD e ABLC). A d-AmB e a L-AmB também apresentaram maior eficácia antifúngica ${ }^{56}$.

Estudos de farmacodinâmica mostraram que d-AmB exibe atividade espécie-específica e concentração-dependente, com concentrações 50\% efetivas $\left(E C_{50}\right)$ variando de 0,10 a $0,12 \mu \mathrm{g} / \mathrm{ml}$ para A. fumigatus; 0,36 a $0,53 \mu \mathrm{g} / \mathrm{ml}$ para $A$. terreus; 0,27 a $\geq 32 \mu \mathrm{g} / \mathrm{ml}$ para $F$. solani; 0,41 a $0,55 \mu \mathrm{g} / \mathrm{ml}$ para F. oxysporum; e 0,97 e 0,65 $\mu \mathrm{g} / \mathrm{ml}$ para S. apiospermum e S. prolificans, respectivamente ${ }^{57,58}$. Em relação à Candida albicans, atividade otimizada similar de AmB pode ser atingida maximizando a relação concentração de pico sobre concentração inibitória 
mínima (CMax/MIC). Ad-AmB também demonstrou atividade inibitória do crescimento prolongada, dose-dependente, mesmo quando seus níveis caíram abaixo do MIC ${ }^{59,60}$.

As formulações lipídicas foram desenvolvidas com o intuito de aumentar o índice terapêutico da $A m B$, permitindo o uso de doses maiores para o tratamento das condições infecciosas. Essas formulações, estruturalmente diversas, diferem em termos de farmacocinética, assim como em relação à concentração tecidual, efeito microbiológico e toxicidade. $\mathrm{Na}$ Tabela 1 podemos observar, resumidamente, as similaridades e diferenças entre as formulações de $A m B$, em relação a algumas características farmacocinéticas e farmacodinâmicas ${ }^{42}$.

\section{EFEITOS ADVERSOS}

A toxicidade da AmB pode ser dividida em efeitos agudos (reações agudas infusionais) e subagudos (toxicidade renal, hematológica e hepática). Reações agudas infusionais são caracterizadas por sintomas como calafrios, febre, náuseas e vômitos, cefaleia, hipotensão, taquicardia e dispneia, que ocorrem durante a administração de d-AmB. Tais reações frequentemente requerem atenção médica e uso de medicamentos, tais como dexclorfeniramina ou meperidina. As reações agudas infusionais relacionadas à administração de d-AmB têm ligação com a liberação de TNF- $\alpha$ e IL-6. Já foram descritos casos de arritmias cardíacas graves durante a administração de $A m B^{7,15,63}$. Estão descritos ainda outros efeitos adversos, como anemia, neutropenia, plaquetopenia (a toxicidade hematológica será abordada nesta revisão), alterações de provas de função hepática e toxicidade cardíaca, essas últimas bem menos comuns ${ }^{15,64}$.

\section{NEFROTOXICIDADE}

A nefrotoxicidade é um evento adverso, bastante conhecido, da terapia com AmB. A perda de função renal é uma complicação relativamente comum, assim como outros eventos, como hipocalemia, hipomagnesemia, acidose metabólica devido a acidose tubular renal distal e poliúria devido a diabetes insipidus nefrogênico ${ }^{13,65,66}$.

Aincidência de nefrotoxicidade pode chegar a níveis entre 49 e 65\%; podendo chegar à perda significativa da função renal, com necessidade de diálise. Fatores de risco para toxicidade por d-AmB incluem sexo masculino, dose elevada do medicamento (acima de $35 \mathrm{mg} / \mathrm{dia}$ ), uso concomitante de diuréticos ou drogas nefrotóxicas, peso corporal acima de $90 \mathrm{~kg} \mathrm{e}$ função renal anormal no baseline ${ }^{13,15,65,67,68}$. A diálise

Tabela 1: Características farmacológicas das formulações de AmB ${ }^{42,56,61,62}$.

\begin{tabular}{|c|c|c|c|}
\hline Característica & L-AmB & ABLC & $\mathrm{d}-\mathrm{AmB}$ \\
\hline $\begin{array}{l}\text { Estrutura } \\
\text { Farmacocinética }\end{array}$ & $\begin{array}{l}\text { Lipossomos esféricos, < 0,080 nm } \\
\text { (dose de } 5 \mathrm{mg} / \mathrm{kg} \text { ) }\end{array}$ & $\begin{array}{l}\text { Complexos tipo fita, 1,6-11 nm } \\
\text { (dose de } 5 \mathrm{mg} / \mathrm{kg} \text { ) }\end{array}$ & $\begin{array}{l}\text { Estrutura micelar, 0-0,35 nm } \\
\text { (dose de 0,6 mg/kg) }\end{array}$ \\
\hline & $\mathrm{CMax}=83 \mathrm{mg} / \mathrm{L}$ & CMax $=1,7 \mathrm{mg} / \mathrm{L}$ & $\mathrm{CMax}=1,1 \mathrm{mg} / \mathrm{L}$ \\
\hline & $\mathrm{AUC}=555 \mathrm{mg} / \mathrm{L}$ & $A \cup C=14 \mathrm{mg} / \mathrm{L}$ & $A \cup C=17,1 \mathrm{mg} / \mathrm{L}$ \\
\hline & $\mathrm{Vd}=0,11 \mathrm{~L} / \mathrm{kg} \cdot \mathrm{h} / \mathrm{mL}$ & $\mathrm{Vd}=131 \mathrm{~L} / \mathrm{kg} \cdot \mathrm{h} / \mathrm{mL}$ & $\mathrm{Vd}=5,1 \mathrm{~L} / \mathrm{kg} \cdot \mathrm{h} / \mathrm{mL}$ \\
\hline & $\mathrm{Cl}=11 \mathrm{mg} / \mathrm{L} / \mathrm{h} / \mathrm{kg}$ & $\mathrm{Cl}=436 \mathrm{mg} / \mathrm{L} / \mathrm{h} / \mathrm{kg}$ & $\mathrm{Cl}=13,1 \mathrm{mg} / \mathrm{L} / \mathrm{h} / \mathrm{kg}$ \\
\hline Toxicidade & $\begin{array}{l}\mathrm{DL}_{50} \text { murinos } \geq 175 \mathrm{mg} / \mathrm{kg} \\
\text { Doses múltiplas em ratos: } \\
20 \mathrm{mg} / \mathrm{kg}=\text { nefrotoxicidade }\end{array}$ & $\begin{array}{l}\mathrm{DL}_{50} \text { murinos } \geq 40 \mathrm{mg} / \mathrm{kg} \\
\text { Doses múltiplas em ratos: } \\
10 \mathrm{mg} / \mathrm{kg}=\text { nefrotoxicidade }\end{array}$ & $\begin{array}{l}\mathrm{DL}_{50} \text { murinos } \geq 4.5 \mathrm{mg} / \mathrm{kg} \\
\text { Doses múltiplas em ratos: } \\
0,025 \mathrm{mg} / \mathrm{kg}=\text { nefrotoxicidade }\end{array}$ \\
\hline $\begin{array}{l}\text { Modo de ação } \\
\text { (carreador) }\end{array}$ & $\begin{array}{l}\text { Direcionamento dos lipossomos } \\
\text { à membrana celular fúngica com } \\
\text { liberação de AmB dentro do fungo }\end{array}$ & $\begin{array}{l}\text { Liberação de AmB dos } \\
\text { complexos no local da } \\
\text { infecção, possivelmente } \\
\text { mediada por macrófagos e } \\
\text { fosfolipases do hospedeiro }\end{array}$ & $\begin{array}{l}\text { Liberação de AmB na corrente } \\
\text { sanguínea }\end{array}$ \\
\hline \multicolumn{4}{|l|}{$\begin{array}{l}\text { Concentração } \\
\text { (em relação à } \\
\text { d-AmB) }\end{array}$} \\
\hline Plasma & Maior & Menor & $\mathrm{n} / \mathrm{a}$ \\
\hline Pulmão & Igual & Maior & $\mathrm{n} / \mathrm{a}$ \\
\hline Fígado & Maior & Maior & $\mathrm{n} / \mathrm{a}$ \\
\hline Rins & Igual & Igual & $\mathrm{n} / \mathrm{a}$ \\
\hline Baço & Maior & Maior & $\mathrm{n} / \mathrm{a}$ \\
\hline Cérebro & Maior & Igual & $\mathrm{n} / \mathrm{a}$ \\
\hline
\end{tabular}


como consequência da perda de função renal foi claramente associada ao aumento na mortalidade ${ }^{16,17}$.

A fisiopatologia da nefrotoxicidade parece envolver dois mecanismos principais: vasoconstrição grave, diminuindo o fluxo sanguíneo renal e, consequentemente, o grau de filtração glomerular (GFR); e uma interação direta da d-AmB com as membrana da células epitelial do rim, causando disfunção tubular. Esses dois mecanismos conjuntamente determinam a perda de função renal ${ }^{13,15}$. Os dois mecanismos interagem em um feedback tubuloglomerular, no qual a concentração baixa de sódio na mácula densa, causada pela disfunção tubular proximal, aumenta a vasoconstrição aferente, reduzindo ainda mais o fluxo sanguíneo renal ${ }^{9,15}$.

Já foi demonstrado que a nefrotoxicidade causada pela AmB é mediada, em nível celular, pelo mecanismo da apoptose. Em um experimento realizado na Alemanha por Varlam e colaboradores, foi estudada a apoptose e sua relação com a nefrotoxicidade pela AmB, em culturas de células e modelos animais. As células do túbulo proximal e as do interstício da medula renal responderam com apoptose a partir de doses terapêuticas de AmB. A aplicação concomitante de fator de crescimento semelhante à insulina-1 recombinante humano (rhIGF-1) foi capaz de inibir essa apoptose in vitro. No modelo animal (ratos Sprague-Dawley) utilizado para verificação, quando da aplicação isolada de AmB, os ratos apresentaram desidratação (evidenciada por gravidade específica mais baixa da urina) e hipocalemia, diferentemente de controles. Na aplicação de AmB em conjunto com rhIGF-1, não houve desidratação e hipocalemia nos animais, da mesma forma que em controles não tratados. Em resumo, o estudo provém evidência de que a apoptose está envolvida diretamente com o processo de nefrotoxicidade associada ao tratamento com AmB, de forma dose-dependente, e que a incidência da apoptose está correlacionada aos sinais e sintomas de nefrotoxicidade ${ }^{24}$. Entretanto, em outro estudo mais recente, no qual foi comparada a nefrotoxicidade de AmB e caspofungina utilizando também ensaios in vitro, não se observou efeito apoptótico com AmB, muito embora tenha sido evidenciado dano celular significativo (e mais pronunciado que caspofungina), causado pela $\mathrm{AmB}^{69}$.

Em uma parcela significativa dos pacientes, d-AmB induz outras alterações: hipocalemia, hipomagnesemia, acidose tubular renal e poliúria devido a diabetes insipidus nefrogênico. Esses efeitos tóxicos devem-se tanto à molécula de AmB em si, mediados pela formação de poros nas células da membrana, como também pelo deoxicolato ${ }^{70}$. A hipocalemia decorre desse aumento da permeabilidade da membrana e acaba por alterar o gradiente de íons existente entre a luz e o citoplasma das células tubulares, prejudicando também a excreção de íons de hidrogênio e causando consequente acidose tubular distal. A hipocalemia é uma complicação frequente da terapia com d-AmB, que pode levar à necessidade de suplementação diária de potássio para a manutenção dos níveis séricos deste íon. A perda de magnésio e a hipomagnesemia também são ocasionadas por essa toxicidade tubular direta. O dano preferencial às células tubulares renais parece ser explicado pela sua exposição ao baixo pH da urina, condição na qual a citotoxicidade é maior e os mecanismos de reparação da membrana ficam comprometidos ${ }^{71}$.

A maior afinidade da d-AmB pelas lipoproteínas do tipo LDL pode explicar a maior nefrotoxicidade observada com essa formulação, em comparação às formulações lipídicas cuja afinidade maior é com $\mathrm{HDL}^{26}$. Os receptores LDL têm uma expressão maior nas células endoteliais do glomérulo renal $\mathrm{e}$, consequentemente, a concentração de d-AmB nos rins é maior do que com as formulações lipídicas ${ }^{26}$.

Para a detecção da nefrotoxicidade, é necessária a vigilância clínica para seus sinais. Suas definições podem variar bastante, mas um aumento de $25 \%$ na creatinina sérica já pode ser considerado como nefrotoxicidade ${ }^{13}$. É importante salientar que o aumento isolado da creatinina, utilizado na quase totalidade dos estudos que avaliaram a nefrotoxicidade da anfotericina, é uma definição de nefrotoxicidade não padronizada e nem validada. Fatores extrarrenais, como massa muscular, volume de distribuição da creatinina, produção endógena da creatinina e função hepática, podem alterar significativamente a creatinina sérica em pacientes gravemente enfermos, e, desta forma, muita cautela deve ser empregada na interpretação de valores isolados de creatinina sérica como um marcador de lesão renal aguda ${ }^{72}$.

Dispõe-se, na atualidade, de critérios objetivos para a definição de nefrotoxicidade, elaborados por grupos de especialidades que estudaram a lesão renal aguda ${ }^{72,73}$. O critério Rifle (Risk, injury, failure, loss of function, endstage renal disease) é a definição mais precisa de nefrotoxicidade na literatura ${ }^{74}$. É um critério já validado para a determinação da incidência de insuficiência renal aguda e de sua estratificação prognóstica em diversas situações clínicas, estando correlacionado com desfechos clínicos duros, como mortalidade. Já o critério Akin (Acute kidney injury network) é uma variação do critério Rifle, e compartilha de muitas de suas vantagens e limitações ${ }^{73}$. A utilização desses critérios nos estudos clínicos é recente, mas tem sido crescente em vários trabalhos nos quais é mensurada incidência de lesão renal aguda, associada ou não a alguma intervenção terapêutica ou diagnóstica. 
A ocorrência de nefrotoxicidade em pacientes tratados com d-AmB pode resultar em prolongamento da hospitalização, aumento de custos relacionados aos cuidados do paciente, além de elevar o risco de óbito. Em uma análise conduzida por Bates et al., em 707 admissões de pacientes tratados com AmB, a insuficiência renal relacionada ao tratamento levou a um aumento, em média, de 8,2 dias no tempo de hospitalização e de 29.823 dólares nos gastos por paciente; além disso, a mortalidade foi mais elevada no grupo de pacientes que desenvolveu insuficiência renal: $54 \%$ versus $16 \%$ no grupo sem disfunção renal. Doses cumulativas mais elevadas de $\mathrm{d}$-AmB foram associadas com chance maior de desenvolver disfunção renal. Nesse estudo, retrospectivo, não houve registros da frequência de uso de medidas nefroprotetoras, como infusão salina ou, ainda, informações sobre a velocidade de infusão utilizada ${ }^{16}$. Uma coorte prospectiva, com dados de pacientes de 20 hospitais diferentes na Europa, avaliou os efeitos da nefrotoxicidade associada ao tratamento com anfotericinas sobre os desfechos clínicos, em especial sobre o tempo de hospitalização. Os pacientes que desenvolveram insuficiência renal na coorte permaneceram, em média, 9 dias a mais no hospital do que o grupo que não apresentou nefrotoxicidade associada ao tratamento com AmB; nefrotoxicidade grave também foi associada a maior mortalidade nesse trabalho ${ }^{17}$. O uso de d-AmB com switch para formulação lipídica (ABLC ou L-AmB) foi comparado ao uso de formulação lipídica somente, em um trabalho retrospectivo que envolveu 408 pacientes. A chance de desenvolver nefrotoxicidade foi 5,69 vezes maior no grupo que fez a terapêutica sequencial d-AmB e anfotericina formulação lipídica, comparado ao grupo que usou somente formulação lipídica. Esses pacientes também permaneceram internados por um período maior no hospital após a terapêutica com anfotericina $(p<0,001)^{75}$.

A infusão contínua de AmB pode ser uma estratégia para diminuir a nefrotoxicidade associada ao uso do medicamento. Está bem documentada a experiência com infusão contínua de d-AmB em pacientes com neutropenia febril, com redução da incidência de nefrotoxicidade associada ao tratamento ${ }^{76}$. Entretanto, o pequeno número de pacientes avaliados em relação à eficácia desta intervenção (ou seja, com infecção fúngica comprovada) faz com que cautela seja recomendada quanto à sua utilização em larga escala na prática clínica ${ }^{76,77}$.

A infusão de um litro de infusão salina por dia, nos pacientes que suportam tal carga hídrica, já está incorporada às práticas clínicas usuais como uma forma de reduzir a nefrotoxicidade associada ao uso de anfotericina $\mathrm{B}^{15,66}$.

\section{TOXICIDADE HEMATOLÓGICA}

Desde os primeiros anos de uso clínico de d-AmB, está bem descrita a ocorrência de anemia associada a esse tratamento ${ }^{10}$. Este efeito adverso é muito comum, estimando-se que ocorra em até $24 \%$ de todos os pacientes tratados; em pacientes que fazem uso da droga por mais de 30 dias, a anemia ocorre em mais de $95 \%$ dos $\operatorname{casos}^{78}$. Em um dos primeiros trabalhos sobre o tema, Brandriss et al. observaram uma queda de pelo menos 11 pontos no hematócrito de $75 \%$ dos pacientes; em $35 \%$ destes foi necessária uma transfusão sanguínea ${ }^{10}$. A anemia poderia influenciar negativamente nos desfechos clínicos, assim como levar a um dispêndio maior de recursos - incluindo-se aí monitorização laboratorial, prolongamento do tempo de hospitalização e uso de hemoderivados. Ainda não há evidências consistentes na literatura sobre as consequências clínicas da anemia induzida pela AmB (tanto na formulação deoxicolato como nas formulações lipídicas), assim como dos custos associados.

Outras alterações hematológicas, como leucopenia e plaquetopenia, também estão descritas. Entretanto, as alterações da série branca e plaquetária parecem ser mais raras, com impacto clínico a ser determinado. A literatura é ainda muito escassa em relação a evidências nesse sentido; um estudo japonês recente encontrou frequência significativa de trombocitopenia associada a doses mais elevadas de L-AmB ${ }^{14,79}$.

A anemia caracteriza-se por ser normocrômica e normocítica, com o número de reticulócitos abaixo do valor normal. Postulou-se inicialmente que ela (traduzida pela queda no hematócrito/hemoglobina) seria decorrente de hemólise ou, ainda, de um estado de deficiência de ferro, induzidos pelo tratamento com AmB. Os estudos iniciais refutaram essas hipóteses com a caracterização da anemia como normocrômica, normocítica e com reticulócitos baixos (o que torna improvável a deficiência de ferro), a demonstração de que a terapia com $A m B$ não aumenta a hemólise (já presente em pacientes com infecções fúngicas) e a não detecção do aumento da eritropoietina quando do nadir da hemoglobina ${ }^{10,78}$. A partir dessas observações, ficou mais evidente que a anemia associada à anfotericina B estaria ligada a um distúrbio na produção de eritropoietina (EPO). Embora isso pudesse estar relacionado à nefrotoxicidade, já que a EPO é produzida pelos rins, os autores desses primeiros trabalhos não encontraram relação entre a diminuição da EPO e a insuficiência renal ${ }^{10,80}$.

Com um conhecimento maior sobre a EPO e seu metabolismo, evidenciou-se que esta é produzida nas células tubulares do rim. Mais especificamente, 
estudos de hibridização detectaram o ácido ribonucleico mensageiro (mRNA) da EPO nas células fibroblásticas do interstício celular próximo aos túbulos proximais do rim. O mRNA da EPO também foi encontrado nas células epiteliais dos túbulos renais proximais ${ }^{81}$.

O mecanismo exato da supressão da produção de EPO pela AmB ainda não foi completamente esclarecido. Foi investigada a possibilidade de que AmB possa ter um efeito inibidor sobre o fator induzível por hipóxia (HIF-1), um conhecido fator de transcrição chave e regulador da produção de EPO. Neste experimento, AmB inativou a atividade de transcrição de HIF-1, promovendo a interação do domínio C-terminal de transativação (CAD) de HIF-1 $\alpha$ e o fator inibidor de HIF-1(FIH), bloqueando o recrutamento do coativador p300 pelo CAD resultando em uma forte inibição da transcrição do gene da EPO. Os níveis de mRNA de EPO foram marcadamente diminuídos pelo tratamento com $A m B$, tanto em cultura de células quanto em rins de ratos. Da mesma forma que nos estudos anteriores, não se confirmou que a nefrotoxicidade estava associada à diminuição na produção de EPO, tendo sido demonstrada a sua supressão mesmo em rins sem alterações microscópicas ou apoptose. O acúmulo da AmB nos rins e, principalmente, sua reabsorção pelos túbulos proximais por sua característica hidrofóbica corroboram o racional de que esse efeito tóxico ocorra em nível molecular nas células produtoras de EPO, impedindo a expressão de mRNA de EPO em resposta à hipóxia ${ }^{81}$.

Em relação à série branca, foi demonstrado que AmB é tóxica aos neutrófilos in vitro; essa toxicidade é revertida pela presença de colesterol LDL ${ }^{12}$. Esta constatação pode ser explicada pela afinidade maior da AmB pelo ergosterol, componente da membrana celular fúngica, do que pelo colesterol. Permanece obscuro o mecanismo pelo qual a droga possa causar trombocitopenia.

Alguns estudos clínicos documentaram a anemia provocada pela terapia com AmB em diferentes cenários. Joly et al., em um estudo de 1996, quando da avaliação do Intralipid - uma combinação de AmB com lipídios em desuso atualmente, chamou a atenção para a ocorrência de anemia. Houve quedas significativas da hemoglobina tanto no grupo do Intralipid quando no grupo deoxicolato, embora esse decréscimo tenha sido maior $(p=0,033)$ no grupo do Intralipid (11,2 g/dL no dia 0 para $7,9 \mathrm{~g} / \mathrm{dL}$ no dia 42) ${ }^{82}$. Sharkey descreveu, em uma comparação de ABLC com d-AmB para o tratamento de meningite criptocócica em pacientes com Aids, maior frequência de anemia em sujeitos que receberam a formulação com deoxicolato $(p<0,05)$. Da mesma forma, pacientes que utilizaram d-AmB foram significativamente mais submetidos à transfusão sanguínea (59\% versus $18 \%, p<0,05)$ do que os pacientes que receberam $A B L C^{47}$. Em um estudo não comparativo de d-AmB em infusão contínua para o tratamento de meningite criptocócica, observou-se uma queda significativa nos níveis médios de hemoglobina (de $11,49 \mathrm{~g} / \mathrm{dL}$ para $7,92 \mathrm{~g} / \mathrm{dL}$ ), sendo que metade dos pacientes apresentou diminuições superiores a $3 \mathrm{~g} / \mathrm{dL}^{77}$.

Shigemi et al. realizaram uma análise de segurança de L-AmB, observando também parâmetros hematológicos em uma pequena coorte retrospectiva. Anemia pós-tratamento foi evidenciada em 10/21 $(47,6 \%)$ pacientes. Já a trombocitopenia foi detectada em 11/19 (57,9\%) dos pacientes. Neste estudo foi possível considerar a dose de L-AmB como um preditor de anemia (OR 3,56; $p<0,05)$ e trombocitopenia (OR 10,01; $p<0,05)$, chamando-se a atenção para esses eventos quando do uso de doses acima de $3,3 \mathrm{mg} / \mathrm{kg} / \mathrm{dia}$ e $3 \mathrm{mg} / \mathrm{kg} / \mathrm{dia}$, respectivamente ${ }^{79}$.

\section{ESTUDOS COMPARATIVOS ENTRE AS FORMULAÇÕES LIPÍDICAS}

Poucos trabalhos efetuaram uma comparação direta, quanto a eficácia e segurança, entre as duas formulações lipídicas mais comumente utilizadas (L-AmB e ABLC). O primeiro estudo realizado com este fim, de Wingard e colaboradores, comparou o perfil de segurança entre essas duas formulações, especialmente no que concerne a reações infusionais agudas e nefrotoxicidade. Foram avaliados 244 pacientes com neutropenia febril, arrolados em três grupos: dois com L-AmB, nas doses de $3 \mathrm{mg} / \mathrm{kg}$ e $5 \mathrm{mg} / \mathrm{kg}$, e outro com ABLC, na dose de $5 \mathrm{mg} / \mathrm{kg}$. Foi definido como nefrotoxicidade um aumento da creatinina de ao menos $100 \%$, em comparação com os valores basais. A exposição média à droga foi de 7,5-8,5 dias; uma parcela significativa de pacientes também recebia outras drogas nefrotóxicas. O uso de infusão salina foi permitido, mas não foi controlado. A frequência de reações infusionais agudas foi significativamente maior no grupo com $A B L C$ do que nos outros dois grupos recebendo L-AmB, inclusive com maior uso de pré-medicação. Foi observada menor nefrotoxicidade nos pacientes utilizando L-AmB, com diferença significativa ( $14,1 \%$ versus $14,8 \%$ versus $42,3 \% ; p<0,001)$. Também foi observada uma frequência menor de descontinuação da droga por toxicidade nos grupos que utilizavam L-AmB ( $32 \%$ versus $13 \% ; p=0,01)$. É importante ressaltar que o protocolo do estudo não previa diminuição ou interrupção de dose; todos os pacientes nos quais, por algum motivo, as drogas em investigação não puderam ser administradas em dose plena foram considerados como tendo abandonado a terapia 
por toxicidade; isto diferiu de outros trabalhos, onde a dose do medicamento poderia ser suspensa, ou mesmo reduzida ${ }^{83}$.

A maioria dos estudos comparando as formulações lipídicas foram observacionais e retrospectivos. O único ensaio clínico randomizado, além do estudo de Wingard citado acima, foi conduzido por Fleming e colaboradores, no MD Anderson Cancer Center (EUA). Esse trabalho, que envolveu um número bastante inferior de pacientes, todos com leucemia ( $n=82 ; 43$ pacientes com ABLC e 39 com L-AmB), definiu nefrotoxicidade como um aumento de $50 \%$ na creatinina. Foi encontrada uma frequência de nefrotoxicidade numericamente maior com ABLC do que com L-AmB ( $40 \%$ versus $28 \%$ ), entretanto sem significância estatística $(p=0,26)$. A quase totalidade dos pacientes nesse estudo estava em uso de outros medicamentos nefrotóxicos ${ }^{84}$. Os outros trabalhos, observacionais, apresentaram resultados conflitantes ou sem significância estatística. Cannon ${ }^{85}$ e Mattiuzzi ${ }^{86}$ demonstraram taxas maiores, porém não significativas, de nefrotoxicidade com L-AmB. Salienta-se que nesse último estudo foi utilizada uma definição distinta de nefrotoxicidade (aumento de $50 \%$ na creatinina sérica). Outros dois estudos retrospectivos evidenciaram maior nefrotoxicidade com ABLC, também sem significância estatística ${ }^{20,87}$. Saliba e colaboradores, em um estudo prospectivo e observacional, com um número pequeno de pacientes, mas que envolveu também pacientes em terapia de resgate para aspergilose (ou seja, pacientes previamente expostos a outros agentes antifúngicos), demonstrou uma tendência a maior nefrotoxicidade com ABLC $(23,3 \%$ com ABLC versus $7,1 \%$ com $L-A m B ; p=0,067)^{20}$. Por fim, uma comparação retrospectiva entre as duas formulações, que incluiu 158 pacientes, também demonstrou uma taxa maior de nefrotoxicidade entre os pacientes que receberam $A B L C$, em comparação à $L-A m B(21,2 \%$ versus $2,8 \% ; p<0,001)^{88}$.

Safdar e colaboradores publicaram recentemente uma metanálise que procurou avaliar especificamente a nefrotoxicidade de duas formulações lipídicas de anfotericina $B, A B L C$ e $L-A m B$. Foram incluídos oito trabalhos nesse estudo, que relata incialmente em separado os resultados de cada trabalho que comparou as duas formulações lipídicas de anfotericina B; após, busca analisá-los em conjunto, utilizando ferramentas estatísticas específicas. A análise de todos os oito trabalhos ( $n=1160$ pacientes) mostrou uma probabilidade maior de disfunção renal no grupo que recebeu ABLC: razão de chances (OR) 1,75 e risco relativo $(R R) 1,55$. No entanto, uma significativa heterogeneidade foi observada entre os estudos. Ao se realizar análises adicionais, que incluíram uma amostra de pacientes em terapia de resgate do estudo de Hachem et al., além de um subgrupo de pacientes do trabalho de Saliba et al. que recebeu tratamento por ao menos 10 dias, ABLC manteve-se mais nefrotóxico que L-AmB, com discreta diminuição do OR e do RR. Novamente, os testes estatísticos mostraram falta de homogeneidade, sugerindo que os resultados deveriam ser interpretados com cautela ${ }^{20}$. Procederam-se, então, novas análises, desta vez excluindo um estudo a cada vez. Foi observado que, excluindo o trabalho de Wingard et al., a probabilidade de nefrotoxicidade era similar entre os pacientes tratados com $A B L C(n=510)$ versus aqueles tratados com L-AmB $(n=406)$; os sete estudos incluídos nessa subanálise ainda não apresentavam homogeneidade pelo teste de Breslow-Day. Utilizando-se a população de pacientes em tratamento de resgate do estudo de Hachem et al., permanecia a ausência de diferença em relação à nefrotoxicidade, com ligeiro aumento na homogeneidade entre os estudos ${ }^{20}$. Uma análise posterior, ainda excluindo o estudo de Wingard et al., adicionando a população de pacientes tratados por dez dias no trabalho de Saliba, continuou demonstrando a ausência de diferença em relação à nefrotoxicidade entre as duas formulações, embora ainda com ausência de homogeneidade entre os estudos. A mesma análise, realizada com os pacientes do estudo de Saliba e com a população de pacientes em resgate do trabalho de Hachem, também não demonstrou diferença significativa na probabilidade de nefrotoxicidade entre as duas formulações ( $n=807$; OR 1,02; RR 1,02) - desta vez, com presença de homogeneidade entre os estudos (teste de heterogeneidade: $p=0,143)^{20}$. O estudo, com sua metodologia passível de crítica, termina por concluir que ABLC e L-AmB são comparáveis em termos de eficácia e segurança, reaquecendo a importância do tema na literatura.

Uma comparação mais recente entre as duas formulações partiu da análise de dados de uma rede de 150 hospitais, gerando dados sobre 327 pacientes (105 com L-AmB e 222 com ABLC). A nefrotoxicidade (definida como um aumento de $100 \%$ do valor da creatinina basal) foi associada, independentemente, com o uso de ABLC (OR 3,48; CI 95\%: 1,05-11,52)

Está bem documentada na literatura a redução na toxicidade com o uso das formulações lipídicas de anfotericina $\mathrm{B}$, como já descrito anteriormente. Diferenças entre elas, no âmbito da nefrotoxicidade, têm sido motivo de intenso debate na comunidade científica. Na metanálise de Safdar, foi demonstrada a ausência de diferença entre as formulações; salientamos a exclusão do estudo de Wingard, que observou essa diferença de forma significativa. Não está claro o motivo da diferença observada 
e de sua magnitude. Aventa-se a possibilidade de diferenças no conceito de nefrotoxicidade utilizado ou, ainda, por ter sido a avaliação da função renal feita, neste estudo, somente até o sétimo dia de tratamento. Em relação a isto, é importante ressaltar um trabalho de Walsh com ABLC, no qual a função renal, após um declínio inicial na primeira semana, mostrou recuperação nas semanas seguintes de tratamento. Desta forma, é possível que a análise de Wingard tenha detectado somente essa piora inicial da função renal com ABLC, potencialmente levando a um pior desempenho da droga no que diz respeito ao risco de nefrotoxicidade ${ }^{19,20,83}$. Por outro lado, um estudo observacional recente envolvendo um grande número de pacientes, que utilizou o mesmo conceito de nefrotoxicidade apresentado na metanálise, evidenciou um risco claramente maior de nefrotoxicidade com $A B L C$ em comparação à L-AmB, conforme já mencionado anteriormente nesta revisão ${ }^{89}$.

\section{USO DE ANFOTERICINA B EM CRIANÇAS}

Existem variações importantes entre adultos e crianças em relação a aspectos de farmacocinética e farmacodinâmica de antifúngicos. Essas variações implicam em considerações importantes quanto à escolha da classe e do regime de administração nos pacientes pediátricos ${ }^{90}$. Embora a evidência seja amplamente disponível para adultos, há limitações no conhecimento sobre o agente ideal para o tratamento de infecções fúngicas invasivas em crianças.

A nefrotoxicidade associada ao tratamento com d-AmB na pediatria ainda é uma questão não completamente esclarecida. Em comparação aos adultos, as crianças tendem a tolerar melhor d-AmB. A incidência de nefrotoxicidade varia entre 15 e 55\%, de forma semelhante aos adultos. Entretanto, a lesão renal reportada em crianças é menos grave, com menor consequência clínica imediata e esperada reversibilidade após a suspensão do tratamento com $A m B^{21,91,92}$. Embora não existam estudos que tenham avaliado a função renal em longo prazo em pacientes pediátricos que receberam tratamento com $\mathrm{AmB}^{21}$, considera-se que se a nefrotoxicidade ocorre nos primeiros anos de vida, pode impactar o bem-estar, em longo prazo, das crianças $^{93}$.

Postula-se que a menor nefrotoxicidade observada em crianças seja advinda de dois fatores: primeiro, a melhor hidratação e ingesta de sódio nos pacientes pediátricos ${ }^{94}$; segundo, na pediatria os pacientes recebem, com a mesma dose de $\mathrm{d}-\mathrm{AmB}(1 \mathrm{mg} / \mathrm{kg} / \mathrm{dia})$, uma exposição menor ao medicamento: em um paciente de $50 \mathrm{~kg}$, isso significa $34 \mathrm{mg} / \mathrm{m}^{2}$; uma criança de $8 \mathrm{~kg}$ recebe, com essa dose, $20 \mathrm{mg} / \mathrm{m}^{221}$.
Mesmo dentro do universo de pacientes pediátricos há uma diferença clara em relação ao risco para nefrotoxicidade. Existe um consenso geral de que a nefrotoxicidade associada ao tratamento do AmB é menor em neonatos, sendo a d-AmB a formulação de escolha nesses pacientes; nos pacientes não neonatos, a decisão de escolher uma formulação lipídica é considerada levando em conta fatores de risco para nefrotoxicidade e, evidentemente, custo e disponibilidade da formulação $0^{21,95}$. Uma preocupação em relação ao uso de formulações lipídicas em neonatos é justamente a menor concentração no tecido renal, considerando a incidência maior de lesões renais, ou no trato urinário, associadas a candidemia nessa população ${ }^{95,96}$.

Comorbidades influenciam também no risco para nefrotoxicidade. Pacientes submetidos a transplante de células-tronco hematopoiéticas (TCTH) são especialmente mais vulneráveis. A coadministração de AmB com outros medicamentos nefrotóxicos, tais como cisplatina e ciclofosfamida, pode aumentar o risco de nefrotoxicidade. Da mesma forma, nefropatia prévia, traduzida por níveis pré-tratamento anormais de creatinina sérica, estão associados com uma incidência maior de nefrotoxicidade ${ }^{91,93}$.

As comparações, quanto ao perfil de segurança entre as diferentes formulações, até o momento, apresentaram resultados contraditórios. Um estudo caso-controle que envolveu 138 pacientes demonstrou nefrotoxicidade menor com ABLC, em comparação à $\mathrm{d}-\mathrm{AmB}(37 \%$ versus $55 \%, p=0,05)$. Salienta-se que, neste estudo, a nefrotoxicidade foi rapidamente reversível, com resolução a níveis normais de creatinina, nas crianças que a apresentaram, em uma semana após a suspensão do tratamento ${ }^{97}$. Outros quatro estudos prospectivos e controlados apresentaram achados discordantes: Prentice ${ }^{92}$ e Walsh ${ }^{18}$ compararam d-AmB com L-AmB, não demonstrando diferença significativa entre as duas formulações, em relação à nefrotoxicidade; por outro lado, Sandler ${ }^{98}$ e White ${ }^{99}$, que compararam d-AmB com $A B C D$, evidenciaram menor toxicidade com esta última $(52,4 \%$ versus $12 \%$; $p=0,003$, e $52,4 \%$ versus $12 \% ; p<0,001$, respectivamente, nos dois estudos). É importante salientar que foi relatada uma incidência muito elevada $(80 \%)$ de reações agudas infusionais com $A B C D^{98}$. A colaboração Cochrane elaborou uma revisão sistemática que incluiu esses quatro estudos. Concluiu-se, a partir da primeira metanálise dentro deste tema, somente com pacientes pediátricos, que formulações lipídicas apresentam menor nefrotoxicidade do que d-AmB. Além disso, a análise do pool de pacientes dos estudos evidenciou que L-AmB está associada a um menor risco de reações agudas infusionais, em comparação 
à d-AmB; e que $A B C D$ está associada a um risco maior de reações agudas infusionais, também em comparação à d-AmB. Ainda quanto à nefrotoxicidade, os autores dessa revisão sistemática fazem a ressalva de que houve significativa heterogeneidade entre os estudos $\left(I^{2}=59 \%\right)^{90}$. Pode-se observar também que a diferença pró-lipídicas vem fundamentalmente dos estudos com $A B C D$, justamente a formulação com risco consistentemente maior para reações agudas infusionais. Ainda, na maioria dos estudos não foi controlada a ingesta de sódio, um fator que pode influenciar no risco para nefrotoxicidade, como já referido ${ }^{94}$

Dessa forma, não há consenso em relação ao benefício das formulações lipídicas (particularmente, L-AmB e ABLC) em pacientes pediátricos. Não existem dados de farmacoeconomia suficientes para justificar ou não, de uma forma generalizada, essas intervenções em crianças. Ainda, não há uma comparação ideal entre o uso de uma formulação lipídica e d-AmB em um ambiente de suplementação de sódio controlado e definições padronizadas de lesão renal aguda. Isso tudo leva para uma decisão caso a caso, onde fatores de risco, como nefropatia prévia, uso de outros medicamentos nefrotóxicos e idade (não neonato $x$ neonato), são decisivos para a escolha da formulação de AmB a ser utilizada no tratamento de infecções fúngicas invasivas.

\section{FARMACOECONOMIA}

Existe uma diferença expressiva no custo de aquisição entre as diferentes formulações de anfotericina B. Enquanto 1 ampola de $50 \mathrm{mg}$ de anfotericina $B$ deoxicolato custa aproximadamente 15 reais, as formulações lipídicas têm um custo consideravelmente mais elevado, no patamar de 1.000 a 1.500 reais por 1 ampola de $50 \mathrm{mg}$ de anfotericina B complexo lipídico ou anfotericina B lipossomal. É importante salientar, entretanto, que o custo de aquisição dos fármacos é apenas uma fração dos custos hospitalares gerais, em que serão adicionados o custo de uma hospitalização mais prolongada, cuidados, exames e procedimentos adicionais que podem vir a ser necessários na ocorrência de toxicidade.

Desde o início do uso clínico das formulações lipídicas de $A m B$ já existe uma preocupação em relação ao custo das formulações, a despeito de suas vantagens em relação à d-AmB ${ }^{100,101}$. Análises farmacoeconômicas já foram desenvolvidas no sentido de avaliar a custo-efetividade das formulações de AmB. Evidentemente todos esses trabalhos baseiam-se no custo do medicamento e custos adicionais da hospitalização, o que pode variar bastante entre diferentes hospitais e de uma forma ainda mais expressiva, entre diferentes países. Cagnoni et al. demonstraram que L-AmB poderia ser mais custo-efetiva do que d-AmB, se o preço por ampola ficasse em torno de 100 dólares (cerca de 300 reais) $)^{102}$. Considerando os preços praticados no mercado americano, um outro estudo (que avaliou três cenários diferentes de preço) demonstrou que $A B L C$ seria mais custo-efetiva do que $L-A m B^{103}$.

Alguns cenários específicos (de população em tratamento ou síndrome clínica tratada) podem estar associados a um perfil de custo-efetividade mais favorável de determinado medicamento. Na aspergilose invasiva, voriconazol foi mais custo-efetivo do que L-AmB em pelo menos dois estudos diferentes de farmacoeconomia ${ }^{104,105}$, e mais custo-efetivo do que d-AmB em outro ${ }^{106}$. Já no tratamento da neutropenia febril, os resultados são contraditórios em relação à comparação entre voriconazol e L-AmB ${ }^{107-109}$, mas o uso de uma equinocandina foi superior à $L-A m B$ em todas as avaliações de farmacoeconomia até o momento ${ }^{110-112}$.

\section{CONCLUSÕES}

A anfotericina B é um antifúngico que possui características que a tornam indispensável dentro do arsenal terapêutico, destacando-se o seu largo espectro antimicrobiano. O conhecimento sobre o seu mecanismo de ação tem avançado: além da formação de poros na membrana celular fúngica, a indução do estresse oxidativo parece ter um papel muito importante em seu efeito antimicrobiano. As novas formulações lipídicas têm características farmacológicas distintas da anfotericina $B$ deoxicolato e, por consequência, um perfil de segurança mais favorável. Embora ainda seja motivo de discussão a existência de diferenças entre as duas formulações lipídicas atualmente disponíveis (L-AmB e ABLC), as evidências apontam para uma incidência menor de efeitos adversos, notadamente nefrotoxicidade, com L-AmB. Outras toxicidades são infrequentes, entretanto a toxicidade hematológica parece ser um fenômeno pouco estudado e com potencial importante para morbidade, considerando a população em que tratamentos com anfotericina B são empregados. Crianças toleram melhor os tratamentos com anfotericina; na pediatria, e particularmente em neonatos, não há consenso sobre o benefício da utilização de formulações lipídicas de AmB. Levando em conta o alto custo associado à aquisição de formulações lipídicas de AmB, são necessários mais estudos de farmacoeconomia avaliando seu uso em situações específicas, e considerando custos hospitalares totais, ajustados ao contexto local. 


\section{REFERÊNCIAS}

1. De Pauw B, Walsh TJ, Donnelly JP, Stevens DA, Edwards JE, Calandra $\mathrm{T}$, et al. Revised definitions of invasive fungal disease from the European Organization for Research and Treatment of Cancer/Invasive Fungal Infections Cooperative Group and the National Institute of Allergy and Infectious Diseases Mycoses Study Group (EORTC/MSG) Consensus Group. Clin Infect Dis. 2008;46(12):1813-21. http://dx.doi. org/10.1086/588660. PMid:18462102.

2. Pappas PG, Alexander BD, Andes DR, Hadley S, Kauffman CA, Freifeld $A$, et al. Invasive fungal infections among organ transplant recipients: results of the Transplant-Associated Infection Surveillance Network (TRANSNET). Clin Infect Dis. 2010;50(8):1101-11. http://dx.doi. org/10.1086/651262. PMid:20218876.

3. Lewis RE, Cahyame-Zuniga $\mathrm{L}$, Leventakos K, Chamilos G, Ben-Ami $\mathrm{R}$, Tamboli $\mathrm{P}$, et al. Epidemiology and sites of involvement of invasive fungal infections in patients with haematological malignancies: a 20-year autopsy study. Mycoses. 2013;56(6):638-45. http:// dx.doi.org/10.1111/myc.12081. PMid:23551865

4. Perfect JR, Schell WA. The new fungal opportunists are coming. Clin Infect Dis. 1996;22(Suppl 2):S112-8. http://dx.doi.org/10.1093/clinids/22. Supplement_2.S112. PMid:8722837.

5. Magill SS, Edwards JR, Bamberg W, Beldavs ZG, Dumyati G, Kainer MA, et al. Multistate pointprevalence survey of health careassociated infections. N Engl J Med. 2014;370(13):1198-208. http://dx.doi. org/10.1056/NEJMoa1306801. PMid:24670166.

6. Kleinberg M. What is the current and future status of conventional amphotericin B? Int J Antimicrob Agents. 2006;27(Suppl 1):126. http://dx.doi.org/10.1016/j. ijantimicag.2006.03.013. PMid:16707251.

7. Lemke A, Kiderlen AF, Kayser O. Amphotericin B. Appl Microbiol Biotechnol. 2005;68(2):151-62. http:// dx.doi.org/10.1007/s00253-005-19559. PMid:15821914.

8. Perfect JR, Dismukes WE, Dromer F, Goldman DL, Graybill JR, Hamill RJ, et al. Clinical practice guidelines for the management of cryptococcal disease: 2010 update by the infectious diseases society of america. Clin Infect Dis. 2010;50(3):291-322. http://dx.doi. org/10.1086/649858. PMid:20047480.

9. Berdichevski RH, Luis LB, Crestana L, Manfro RC. Amphotericin B-related nephrotoxicity in low-risk patients. Braz J Infect Dis. 2006;10(2):949. http://dx.doi.org/10.1590/ S1413-86702006000200005. PMid:16878259.

10. Brandriss MW, Wolff SM, Moores R, Stohlman F JR. Anemia induced by amphotericin B. JAMA. 1964;189(9):6636. http://dx.doi.org/10.1001/ jama.1964.03070090013003. PMid:14172265.

11. Chan CS, Tuazon CU, Lessin LS. Amphotericin-B-induced thrombocytopenia. Ann Intern Med. 1982;96(3):332-3. http://dx.doi. org/10.7326/0003-4819-96-3-332. PMid:6949486.

12. Chunn CJ, Starr PR, Gilbert DN. Neutrophil toxicity of amphotericin B. Antimicrob Agents Chemother. 1977;12(2):226-30. http://dx.doi. org/10.1128/AAC.12.2.226 PMid:900919.

13. Deray G. Amphotericin B nephrotoxicity. J Antimicrob Chemother. 2002;49(Suppl 1):3741. http://dx.doi.org/10.1093/jac/49. suppl_1.37. PMid:11801579.

14. Stein JB, Tolle SW. Episodic leukopenia associated with amphotericin B. South Med J. 1983;76(3):409-10. http://dx.doi. org/10.1097/00007611-19830300000037. PMid:6828914.

15. Laniado-Laborín R, Cabrales-Vargas MN. Amphotericin B: side effects and toxicity. Rev Iberoam Micol. 2009;26(4):223-7. http://dx.doi. org/10.1016/j.riam.2009.06.003. PMid:19836985.

16. Bates DW, Su L, Yu DT, Chertow GM, Seger DL, Gomes DR, et al. Mortality and costs of acute renal failure associated with amphotericin B therapy. Clin Infect Dis. 2001;32(5):686-93. http://dx.doi. org/10.1086/319211. PMid:11229835.

17. Ullmann AJ, Sanz MA, Tramarin A, Barnes RA, Wu W, Gerlach BA, et al.
Prospective study of amphotericin B formulations in immunocompromised patients in 4 European countries. Clin Infect Dis. 2006;43(4):e29-38. http://dx.doi.org/10.1086/505969. PMid:16838223.

18. Walsh TJ, Finberg RW, Arndt C, Hiemenz J, Schwartz C, Bodensteiner $D$, et al. Liposomal amphotericin B for empirical therapy in patients with persistent fever and neutropenia. N Engl J Med. 1999;340(10):76471. http://dx.doi.org/10.1056/ NEJM199903113401004. PMid:10072411.

19. Walsh TJ, Hiemenz JW, Seibel NL, Perfect JR, Horwith G, Lee L, et al. Amphotericin B lipid complex for invasive fungal infections: analysis of safety and efficacy in 556 cases. Clin Infect Dis. 1998;26(6):1383-96 http://dx.doi.org/10.1086/516353. PMid:9636868.

20. Safdar A, Ma J, Saliba F, Dupont $B$, Wingard JR, Hachem RY, et al. Drug-induced nephrotoxicity caused by amphotericin B lipid complex and liposomal amphotericin B: a review and meta-analysis. Medicine (Baltimore). 2010;89(4):23644. http://dx.doi.org/10.1097/ MD.0b013e3181e9441b. PMid:20616663.

21. Bes DF, Rosanova MT, Sberna $\mathrm{N}$, Arrizurieta E. Deoxycholate amphotericin $B$ and nephrotoxicity in the pediatric setting. Pediatr Infect Dis J. 2014;33(8):e198-206. http://dx.doi. org/10.1097/INF.0000000000000299. PMid:24618932.

22. Mesa-Arango AC, Scorzoni L, Zaragoza O. It only takes one to do many jobs: Amphotericin $B$ as antifungal and immunomodulatory drug. Front Microbiol. 2012;3(286):110. http://dx.doi.org/10.3389/ fmicb.2012.00286. PMid:23024638.

23. Ben-Ami R, Lewis RE, Kontoyiannis DP. Immunocompromised hosts: immunopharmacology of modern antifungals. Clin Infect Dis. 2008;47(2):226-35. http://dx.doi. org/10.1086/589290. PMid:18540822.

24. Varlam DE, Siddiq MM, Parton LA, Rüssmann $\mathrm{H}$. Apoptosis contributes to amphotericin B-induced nephrotoxicity. Antimicrob Agents Chemother. 2001;45(3):679-85. http:// dx.doi.org/10.1128/AAC.45.3.679685.2001. PMid:11181342. 
25. Adler-Moore J, Proffitt RT. AmBisome: liposomal formulation, structure, mechanism of action and pre-clinical experience. J Antimicrob Chemother. 2002;49(Suppl 1):21-30. http://dx.doi. org/10.1093/jac/49.suppl_1.21. PMid:11801577.

26. Walsh TJ, Goodman JL, Pappas P, Bekersky I, Buell DN, Roden $M$, et al. Safety, tolerance, and pharmacokinetics of highdose liposomal amphotericin B (AmBisome) in patients infected with Aspergillus species and other filamentous fungi: maximum tolerated dose study. Antimicrob Agents Chemother. 2001;45(12):348796. http://dx.doi.org/10.1128/ AAC.45.12.3487-3496.2001. PMid:11709329.

27. Adler-Moore J. AmBisome targeting to fungal infections. Bone Marrow Transplant. 1994;14(Suppl 5):S3-7. PMid:7703928.

28. Adler-Moore JP, Chiang SM, Satorius A, Guerra D, McAndrews B, McManus EJ, et al. Treatment of murine candidosis and cryptococcosis with a unilamellar liposomal amphotericin B formulation (AmBisome). J Antimicrob Chemother. 1991;28(Suppl B):6371. http://dx.doi.org/10.1093/jac/28. suppl_B.63. PMid:1778893.

29. Lee JW, Amantea MA, Francis PA, Navarro EE, Bacher J, Pizzo PA, et al. Pharmacokinetics and safety of a unilamellar liposomal formulation of amphotericin $B$ (AmBisome) in rabbits. Antimicrob Agents Chemother. 1994;38(4):7138. http://dx.doi.org/10.1128/ AAC.38.4.713. PMid:8031034.

30. Meunier F, Prentice HG, Ringdén O. Liposomal amphotericin B (AmBisome): safety data from a phase II/III clinical trial. J Antimicrob Chemother. 1991;28(Suppl B):8391. http://dx.doi.org/10.1093/jac/28. suppl_B.83. PMid:1778895.

31. Proffitt RT, Satorius A, Chiang SM, Sullivan L, Adler-Moore JP. Pharmacology and toxicology of a liposomal formulation of amphotericin B (AmBisome) in rodents. J Antimicrob Chemother. 1991;28(Suppl B):49-61. http://dx.doi. org/10.1093/jac/28.suppl_B.49. PMid:1778892.

32. Chopra R, Blair S, Strang J, Cervi P, Patterson KG, Goldstone AH. Liposomal amphotericin B (AmBisome) in the treatment of fungal infections in neutropenic patients. J Antimicrob Chemother. 1991;28(Suppl B):93-104. http:// dx.doi.org/10.1093/jac/28.suppl_B.93. PMid:1778896.

33. Coker RJ, Murphy SM, Harris JR. Experience with liposomal amphotericin B (AmBisome) in cryptococcal meningitis in AIDS. J Antimicrob Chemother. 1991;28(Suppl B):105-9. http://dx.doi. org/10.1093/jac/28.suppl_B.105. PMid:1778887.

34. Croft SL, Davidson RN, Thornton EA. Liposomal amphotericin B in the treatment of visceral leishmaniasis. $J$ Antimicrob Chemother. 1991;28(Suppl B):111-8. http://dx.doi. org/10.1093/jac/28.suppl_B.111. PMid:1778888.

35. Leenders AC, Daenen S, Jansen RL, Hop WC, Lowenberg B Wijermans PW, et al. Liposomal amphotericin B compared with amphotericin $B$ deoxycholate in the treatment of documented and suspected neutropenia-associated invasive fungal infections. $\mathrm{Br} \mathrm{J}$ Haematol. 1998;103(1):205-12. http://dx.doi.org/10.1046/j.13652141.1998.00944.x. PMid:9792309.

36. Ringdén $\mathrm{O}$, Meunier F, Tollemar J, Ricci P, Tura S, Kuse E, et al. Efficacy of amphotericin $B$ encapsulated in liposomes (AmBisome) in the treatment of invasive fungal infections in immunocompromised patients. J Antimicrob Chemother. 1991;28(Suppl B):73-82. http://dx.doi. org/10.1093/jac/28.suppl_B.73. PMid:1778894.

37. Bern C, Adler-Moore J, Berenguer J, Boelaert M, den Boer M, Davidson $\mathrm{RN}$, et al. Liposomal amphotericin $B$ for the treatment of visceral leishmaniasis. Clin Infect Dis. 2006;43(7):917-24. http://dx.doi. org/10.1086/507530. PMid:16941377.

38. Cornely OA, Maertens J, Bresnik M, Ebrahimi R, Ullmann AJ, Bouza E, et al, and the AmBiLoad Trial Study Group. Liposomal amphotericin $B$ as initial therapy for invasive mold infection: a randomized trial comparing a high-loading dose regimen with standard dosing (AmBiLoad trial). Clin Infect Dis. 2007;44(10):1289-97. http://dx.doi. org/10.1086/514341. PMid:17443465.

39. Johnson PC, Wheat LJ, Cloud GA, Goldman M, Lancaster D, Bamberger DM, et al. Safety and efficacy of liposomal amphotericin B compared with conventional amphotericin B for induction therapy of histoplasmosis in patients with AIDS. Ann Intern Med. 2002;137(2):105-9. http:// dx.doi.org/10.7326/0003-4819-137-2200207160-00008. PMid:12118965.

40. Sundar S, Chakravarty J, Agarwal D, Rai M, Murray HW. Single-dose liposomal amphotericin B for visceral leishmaniasis in India. N Engl J Med. 2010;362(6):504-12. http://dx.doi. org/10.1056/NEJMoa0903627. PMid:20147716.

41. Levine SJ, Walsh TJ, Martinez A, Eichacker PQ, Lopez-Berestein G, Natanson C. Cardiopulmonary toxicity after liposomal amphotericin $B$ infusion. Ann Intern Med. 1991;114(8):664-6. http://dx.doi. org/10.7326/0003-4819-114-8-664. PMid:2003714.

42. Adler-Moore JP, Proffitt RT. Amphotericin $B$ lipid preparations: what are the differences? Clin Microbiol Infect. 2008;14(Suppl 4):2536. http://dx.doi.org/10.1111/j.14690691.2008.01979.x. PMid:18430127.

43. Janoff AS, Boni LT, Popescu MC, Minchey SR, Cullis PR, Madden $T D$, et al. Unusual lipid structures selectively reduce the toxicity of amphotericin B. Proc Natl Acad Sci USA. 1988;85(16):6122-6. http:// dx.doi.org/10.1073/pnas.85.16.6122. PMid:3413081.

44. Hamill RJ. Amphotericin B formulations: a comparative review of efficacy and toxicity. Drugs. 2013;73(9):919-34. http://dx.doi. org/10.1007/s40265-013-0069-4. PMid:23729001.

45. Wingard JR. Efficacy of amphotericin $B$ lipid complex injection (ABLC) in bone marrow transplant recipients with life-threatening systemic mycoses. Bone Marrow Transplant. 1997;19(4):343-7. http://dx.doi. org/10.1038/sj.bmt.1700664. PMid:9051244.

46. Anaissie E, White M, Uzun O, Singer C, Bodey GP, Matzke D, LopezBerestein, G. Amphotericin B lipid complex versus amphotericin $B$ for treatment of invasive candidiasis: a prospective, randomized, multicenter trial [abstract LM21]. In: Interscience Conference on Antimicrobial Agents and Chemotherapy (ICAAC); 1995; San Francisco: American Society of Microbiology.

47. Sharkey PK, Graybill JR, Johnson ES, Hausrath SG, Pollard RB, Kolokathis A, et al. Amphotericin 
B lipid complex compared with amphotericin B in the treatment of cryptococcal meningitis in patients with AIDS. Clin Infect Dis. 1996;22(2):308-14. http://dx.doi. org/10.1093/clinids/22.2.315. PMid:8838189.

48. Subirà $M$, Martino $R$, Gómez $L$, Martí JM, Estany C, Sierra J. Lowdose amphotericin $B$ lipid complex vs. conventional amphotericin B for empirical antifungal therapy of neutropenic fever in patients with hematologic malignancies--a randomized, controlled trial. Eur J Haematol. 2004;72(5):342-7. http://dx.doi.org/10.1111/j.16000609.2004.00239.x. PMid:15059069.

49. Aguado JM, Lumbreras C, González-Vidal D. Assessment of nephrotoxicity in patients receiving amphotericin B lipid complex: a pharmacosurveillance study in Spain. Clin Microbiol Infect. 2004;10(9):78590. http://dx.doi.org/10.1111/j.1198743X.2004.00963.x. PMid:15355408.

50. Martino R. Efficacy, safety and cost-effectiveness of Amphotericin B Lipid Complex (ABLC): a review of the literature. Curr Med Res Opin. 2004;20(4):485-504. http://dx.doi. org/10.1185/030079904125003179. PMid:15119986.

51. Wingard JR. Lipid formulations of amphotericins: are you a lumper or a splitter? Clin Infect Dis. 2002;35(7):891-5. http://dx.doi. org/10.1086/342563. PMid:12228829.

52. Lewis RE, Wiederhold NP. The solubility ceiling: a rationale for continuous infusion amphotericin B therapy? Clin Infect Dis. 2003;37(6):871-2. http://dx.doi. org/10.1086/377279. PMid:12955666.

53. Bindschadler DD, Bennett JE. A pharmacologic guide to the clinical use of amphotericin B. J Infect Dis. 1969;120(4):427-36. http://dx.doi. org/10.1093/infdis/120.4.427. PMid:5394441.

54. Bekersky I, Fielding RM, Dressler DE, Lee JW, Buell DN, Walsh TJ. Pharmacokinetics, excretion, and mass balance of liposomal amphotericin B (AmBisome) and amphotericin $B$ deoxycholate in humans. Antimicrob Agents Chemother. 2002;46(3):828-33. http:// dx.doi.org/10.1128/AAC.46.3.828833.2002. PMid:11850268.

55. Andes D. Pharmacokinetics and pharmacodynamics of antifungals. Infect Dis Clin North Am.
2006;20(3):679-97. http://dx.doi. org/10.1016/j.idc.2006.06.007. PMid:16984875.

56. Groll $A H$, Giri N, Petraitis V, Petraitiene R, Candelario M, Bacher JS, et al. Comparative efficacy and distribution of lipid formulations of amphotericin B in experimental Candida albicans infection of the central nervous system. $J$ Infect Dis. 2000;182(1):274-82. http://dx.doi. org/10.1086/315643. PMid:10882607.

57. Wiederhold NP, Tam VH, Chi J, Prince RA, Kontoyiannis DP, Lewis RE. Pharmacodynamic activity of amphotericin B deoxycholate is associated with peak plasma concentrations in a neutropenic murine model of invasive pulmonary aspergillosis. Antimicrob Agents Chemother. 2006;50(2):469-73. http:// dx.doi.org/10.1128/AAC.50.2.469473.2006. PMid:16436698.

58. Lewis RE, Wiederhold NP, Klepser ME. In vitro pharmacodynamics of amphotericin B, itraconazole, and voriconazole against Aspergillus, Fusarium, and Scedosporium spp. Antimicrob Agents Chemother. 2005;49(3):945-51. http://dx.doi. org/10.1128/AAC.49.3.945-951.2005. PMid:15728887.

59. Burgess DS, Hastings RW, Summers KK, Hardin TC, Rinaldi MG. Pharmacodynamics of fluconazole, itraconazole, and amphotericin B against Candida albicans. Diagn Microbiol Infect Dis. 2000;36(1):13-8. http://dx.doi.org/10.1016/S07328893(99)00097-8. PMid:10744363.

60. Andes D, Stamsted T, Conklin R. Pharmacodynamics of amphotericin $B$ in a neutropenic-mouse disseminated-candidiasis model. Antimicrob Agents Chemother. 2001;45(3):922-6. http://dx.doi. org/10.1128/AAC.45.3.922-926.2001. PMid:11181381.

61. Cifani C, Costantino S, Massi M, Berrino L. Commercially available lipid formulations of amphotericin b: are they bioequivalent and therapeutically equivalent? Acta Biomed. 2012;83(2):154-63. PMid:23393924.

62. Tiphine $M$, Letscher-Bru $\mathrm{V}$, Herbrecht R. Amphotericin $B$ and its new formulations: pharmacologic characteristics, clinical efficacy, and tolerability. Transpl Infect Dis. 1999;1(4):27383. http://dx.doi.org/10.1034/ j.1399-3062.1999.010406.x. PMid:11428998.

63. Ellis ME, al-Hokail AA, Clink HM, Padmos MA, Ernst P, Spence $D G$, et al. Double-blind randomized study of the effect of infusion rates on toxicity of amphotericin B. Antimicrob Agents Chemother. 1992;36(1):1729. http://dx.doi.org/10.1128/ AAC.36.1.172. PMid:1590686.

64. Moyssakis I, Vassilakopoulos TP, Sipsas NV, Perakis A, Petrou A, Kosmas N, et al. Reversible dilated cardiomyopathy associated with amphotericin B treatment. Int $\mathrm{J}$ Antimicrob Agents. 2005;25(5):4447. http://dx.doi.org/10.1016/j. ijantimicag.2005.02.015. PMid:15848302.

65. Bates DW, Su L, Yu DT, Chertow GM, Seger DL, Gomes DR, et al. Correlates of acute renal failure in patients receiving parenteral amphotericin B. Kidney Int. 2001;60(4):1452-9. http:// dx.doi.org/10.1046/j.15231755.2001.00948.x. PMid:11576359.

66. Branch RA. Prevention of amphotericin B-induced renal impairment. A review on the use of sodium supplementation. Arch Intern Med. 1988;148(11):238994. http://dx.doi.org/10.1001/ archinte.1988.00380110049010. PMid:3056312.

67. Pasqualotto AC. Amphotericin B: the higher the dose, the higher the toxicity. Clin Infect Dis. 2008;47(8):1110-1111. http://dx.doi. org/10.1086/592117. PMid:18800934.

68. Harbarth S, Pestotnik SL, Lloyd JF, Burke JP, Samore MH. The epidemiology of nephrotoxicity associated with conventional amphotericin B therapy. Am J Med. 2001;111(7):528-34. http://dx.doi. org/10.1016/S0002-9343(01)009287. PMid:11705428.

69. Wegner B, Baer P, Gauer S, Oremek G, Hauser IA, Geiger H. Caspofungin is less nephrotoxic than amphotericin $B$ in vitro and predominantly damages distal renal tubular cells. Nephrol Dial Transplant. 2005;20(10):2071-9. http://dx.doi.org/10.1093/ndt/gfh948. PMid:15998654.

70. Zager RA, Bredl CR, Schimpf BA Direct amphotericin B-mediated tubular toxicity: assessments of selected cytoprotective agents. Kidney Int. 1992;41(6):1588-94. 
http://dx.doi.org/10.1038/ki.1992.229. PMid:1501413.

71. Walev I, Bhakdi S. Possible reason for preferential damage to renal tubular epithelial cells evoked by amphotericin B. Antimicrob Agents Chemother. 1996;40(5):1116-20. PMid:8723450.

72. Venkataraman R, Kellum JA. Defining acute renal failure: the RIFLE criteria. J Intensive Care Med. 2007;22(4):187-93. http://dx.doi. org/10.1177/0885066607299510. PMid:17712054

73. International Society of Nephrology. KDIGO Clinical Practice Guideline for Acute Kidney Injury. Kidney Int. 2012;2(Suppl 1):1-138.

74. Fujii T, Uchino S, Takinami M, Bellomo R. Validation of the Kidney Disease Improving Global Outcomes criteria for AKI and comparison of three criteria in hospitalized patients. Clin J Am Soc Nephrol. 2014;9(5):848-54. http://dx.doi. org/10.2215/CJN.09530913. PMid:24578334

75. Wade RL, Chaudhari P, Natoli JL, Taylor RJ, Nathanson BH, Horn D. Comparison of adverse events and hospital length of stay associated with various amphotericin $B$ formulations: sequential conventional amphotericin b/lipid versus lipid-only therapy for the treatment of invasive fungal infections in hospitalized patients. $P T$. 2013;38(5):278-87. PMid:23946621.

76. Falci DR, dos Santos RP, Wirth F, Goldani LZ. Continuous infusion of amphotericin B deoxycholate: an innovative, low-cost strategy in antifungal treatment.

Mycoses. 2011;54(2):91-8.

http://dx.doi.org/10.1111/j.14390507.2009.01805.x. PMid:19878457.

77. Falci DR, Lunardi LW, Ramos CG, Bay MB, Aquino VR, Goldani LZ. Continuous infusion of amphotericin $B$ deoxycholate in the treatment of cryptococcal meningoencephalitis: analysis of safety and fungicidal activity. Clin Infect Dis. 2010;50(5):e26-9. http://dx.doi. org/10.1086/650489. PMid:20121575.

78. Lin AC, Goldwasser E, Bernard EM, Chapman SW. Amphotericin B blunts erythropoietin response to anemia. $J$ Infect Dis. 1990;161(2):348-51. http:// dx.doi.org/10.1093/infdis/161.2.348. PMid:2299214.

79. Shigemi A, Matsumoto K, Ikawa K, Yaji K, Shimodozono Y, Morikawa
$\mathrm{N}$, et al. Safety analysis of liposomal amphotericin B in adult patients: anaemia, thrombocytopenia, nephrotoxicity, hepatotoxicity and hypokalaemia. Int J Antimicrob Agents. 2011;38(5):417-20. http://dx.doi.org/10.1016/j. ijantimicag.2011.07.004. PMid:21885259.

80. MacGregor RR, Bennett JE, Erslev AJ. Erythropoietin concentration in amphotericin B-induced anemia. Antimicrob Agents Chemother. 1978;14(2):270-3. http://dx.doi. org/10.1128/AAC.14.2.270. PMid:697352.

81. Yeo EJ, Ryu JH, Cho YS, Chun YS, Huang LE, Kim MS, et al. Amphotericin B blunts erythropoietin response to hypoxia by reinforcing $\mathrm{FlH}$-mediated repression of HIF-1. Blood. 2006;107(3):916-23. http://dx.doi. org/10.1182/blood-2005-06-2564. PMid:16189267.

82. Joly V, Aubry P, Ndayiragide A, Carrière I, Kawa E, Mlika-Cabanne $\mathrm{N}$, et al. Randomized comparison of amphotericin B deoxycholate dissolved in dextrose or Intralipid for the treatment of AIDS-associated cryptococcal meningitis. Clin Infect Dis. 1996;23(3):556-62. http:// dx.doi.org/10.1093/clinids/23.3.556. PMid:8879780.

83. Wingard JR, White MH, Anaissie E, Raffalli J, Goodman J, Arrieta A. A randomized, double-blind comparative trial evaluating the safety of liposomal amphotericin $B$ versus amphotericin $B$ lipid complex in the empirical treatment of febrile neutropenia. L Amph/ABLC Collaborative Study Group. Clin Infect Dis. 2000;31(5):1155-63. http://dx.doi. org/10.1086/317451. PMid:11073745.

84. Fleming RV, Kantarjian HM, Husni R, Rolston K, Lim J, Raad I, et al. Comparison of amphotericin $B$ lipid complex (ABLC) vs. ambisome in the treatment of suspected or documented fungal infections in patients with leukemia. Leuk Lymphoma. 2001;40(5-6):511-20. http://dx.doi. org/10.3109/10428190109097650. PMid:11426524.

85. Cannon JP, Garey KW, Danziger $\mathrm{LH}$. A prospective and retrospective analysis of the nephrotoxicity and efficacy of lipid-based amphotericin B formulations. Pharmacotherapy. 2001;21(9):1107-14. http://dx.doi. org/10.1592/phco.21.13.1107.34613. PMid:11560200.

86. Mattiuzzi GN, Kantarjian H, Faderl S, Lim J, Kontoyiannis D, Thomas $\mathrm{D}$, et al. Amphotericin B lipid complex as prophylaxis of invasive fungal infections in patients with acute myelogenous leukemia and myelodysplastic syndrome undergoing induction chemotherapy. Cancer. 2004;100(3):581-9. http:// dx.doi.org/10.1002/cncr.11936. PMid:14745876.

87. Malani PN, Depestel DD, Riddell J 4TH, Bickley S, Klein LR, Kauffman CA. Experience with community-based amphotericin B infusion therapy. Pharmacotherapy. 2005;25(5):690-7. http://dx.doi. org/10.1592/phco.25.5.690.63591. PMid:15899731.

88. Hachem RY, Boktour MR, Hanna HA, Husni RN, Torres $\mathrm{HA}$, Afif C, et al. Amphotericin B lipid complex versus liposomal amphotericin B monotherapy for invasive aspergillosis in patients with hematologic malignancy. Cancer. 2008;112(6):1282-7. http:// dx.doi.org/10.1002/cncr.23311. PMid:18224662.

89. Wade RL, Chaudhari P, Natoli JL, Taylor RJ, Nathanson BH, Horn DL. Nephrotoxicity and other adverse events among inpatients receiving liposomal amphotericin B or amphotericin B lipid complex. Diagn Microbiol Infect Dis. 2013;76(3):3617. http://dx.doi.org/10.1016/j. diagmicrobio.2013.04.001. PMid:23774005.

90. Blyth CC, Hale K, Palasanthiran P, O'Brien T, Bennett MH. Antifungal therapy in infants and children with proven, probable or suspected invasive fungal infections. Cochrane Database Syst Rev. 2010;(2):CD006343. PMid:20166083.

91. Goldman RD, Ong M, Wolpin J, Parshuram C, Koren G, Doyle J. Pharmacological risk factors for amphotericin B nephrotoxicity in children. J Clin Pharmacol. 2007;47(8):1049-54. http://dx.doi. org/10.1177/0091270007301799. PMid:17525169.

92. Prentice HG, Hann IM, Herbrecht R, Aoun M, Kvaloy S, Catovsky $\mathrm{D}$, et al. A randomized comparison of liposomal versus conventional amphotericin B for the treatment of pyrexia of unknown origin 
in neutropenic patients. $\mathrm{Br} \mathrm{J}$ Haematol. 1997;98(3):711-

8. http://dx.doi.org/10.1046/ j.1365-2141.1997.2473063.x. PMid:9332329.

93. Goldman RD, Koren G. Amphotericin $B$ nephrotoxicity in children. J Pediatr Hematol Oncol. 2004;26(7):421-6. http://dx.doi.org/10.1097/00043426200407000-00004. PMid:15218415.

94. Turcu R, Patterson MJ, Omar $\mathrm{S}$. Influence of sodium intake on Amphotericin B-induced nephrotoxicity among extremely premature infants. Pediatr Nephrol. 2009;24(3):497-505. http://dx.doi. org/10.1007/s00467-008-1050-4. PMid:19082636.

95. Turkova A, Roilides E, Sharland M. Amphotericin B in neonates: deoxycholate or lipid formulation as first-line therapy - is there a 'right' choice? Curr Opin Infect Dis. 2011;24(2):16371. http://dx.doi.org/10.1097/ QCO.0b013e328343614e. PMid:21301335.

96. Cetin H, Yalaz M, Akisu M, Hilmioglu S, Metin D, Kultursay $\mathrm{N}$. The efficacy of two different lipid-based amphotericin B in neonatal Candida septicemia. Pediatr Int. 2005;47(6):676-80. http://dx.doi.org/10.1111/j.1442200x.2005.02135.x. PMid:16354223.

97. Dutta A, Palazzi DL. Risk factors of amphotericin B toxicty in the nonneonatal pediatric population. Pediatr Infect Dis J. 2012;31(9):9104. http://dx.doi.org/10.1097/ INF.0b013e31825d649a. PMid:22581225.

98. Sandler ES, Mustafa MM, Tkaczewski I, Graham ML, Morrison VA, Green M, et al. Use of amphotericin B colloidal dispersion in children. J Pediatr Hematol Oncol. 2000;22(3):242-6. http://dx.doi. org/10.1097/00043426-20000500000009. PMid: 10864055.

99. White MH, Bowden RA, Sandler ES, Graham ML, Noskin GA, Wingard $\mathrm{JR}$, et al. Randomized, doubleblind clinical trial of amphotericin B colloidal dispersion vs. amphotericin $B$ in the empirical treatment of fever and neutropenia. Clin Infect Dis. 1998;27(2):296-302. http://dx.doi. org/10.1086/514672. PMid:9709879.
100. Spellberg B, Witt MD, Beck CK. Amphotericin B: is a lipidformulation gold standard feasible? Clin Infect Dis. 2004;38(2):304-5, author reply 306-7. http://dx.doi. org/10.1086/380844. PMid:14699470.

101. Tollemar J, Ringdén O. Lipid formulations of amphotericin B. Less toxicity but at what economic cost? Drug Saf. 1995;13(4):207-18. http://dx.doi.org/10.2165/00002018199513040-00001. PMid:8573294.

102. Cagnoni PJ, Walsh TJ, Prendergast MM, Bodensteiner D, Hiemenz S, Greenberg RN, et al. Pharmacoeconomic analysis of liposomal amphotericin $B$ versus conventional amphotericin $B$ in the empirical treatment of persistently febrile neutropenic patients. $J$ Clin Oncol. 2000;18(12):2476-83. PMid:10856108.

103. Kuti JL, Kotapati S, Williams $P$, Capitano B, Nightingale $\mathrm{CH}$, Nicolau DP. Pharmacoeconomic analysis of amphotericin B lipid complex versus liposomal amphotericin $B$ in the treatment of fungal infections. Pharmacoeconomics. 2004;22(5):301-10. http://dx.doi. org/10.2165/00019053-20042205000004. PMid:15061680.

104. Luong ML, Husain S, Rotstein C. Pharmacoeconomic assessment of therapy for invasive aspergillosis. Mycoses. 2013;56(3):338-49. http:// dx.doi.org/10.1111/myc.12036. PMid:23311989.

105. Ostermann H, Solano C, Jarque I, Garcia-Vidal C, Gao X, Barrueta $\mathrm{JA}$, et al. Cost analysis of voriconazole versus liposomal amphotericin B for primary therapy of invasive aspergillosis among patients with haematological disorders in Germany and Spain. BMC Pharmacol Toxicol. 2014;15(52):1-8. http:// dx.doi.org/10.1186/2050-6511-15-52. PMid:25253630.

106. Grau Cerrato S, Mateu-de Antonio $\mathrm{J}$, Soto Álvarez J, Muñoz Jareño MA, Salas Sánchez E, Marín-Casino $M$, et al. Evaluación económica del uso de voriconazol versus anfotericina $B$ en el tratamiento de la aspergilosis invasiva. Farm Hosp. 2005;29(1):5-10. http://dx.doi. org/10.1016/S1130-6343(05)73629-1. PMid:15773796.
107. Al-Badriyeh D, Liew D, Stewart K, Kong DC. Cost-effectiveness evaluation of voriconazole versus liposomal amphotericin $B$ as empirical therapy for febrile neutropenia in Australia. J Antimicrob Chemother. 2009;63(1):197-208. http://dx.doi.org/10.1093/jac/dkn459. PMid:19001450.

108. Collins CD, Stuntebeck ER, DePestel DD, Stevenson JG. Pharmacoeconomic analysis of liposomal amphotericin B versus voriconazole for empirical treatment of febrile neutropenia. Clin Drug Investig. 2007;27(4):233-41. http:// dx.doi.org/10.2165/00044011200727040-00002. PMid:17358095.

109. Turner SJ, Senol E, Kara A, AlBadriyeh D, Dinleyici EC, Kong DC. Pharmacoeconomic evaluation of voriconazole vs. liposomal amphotericin B in empiric treatment of invasive fungal infections in Turkey. BMC Infect Dis. 2013;13(560):1-9. http://dx.doi.org/10.1186/1471-233413-560. PMid:24279677.

110. Neoh CF, Liew D, Slavin MA, Marriott D, Chen SC, Morrissey O, et al. Economic evaluation of micafungin vs. liposomal amphotericin B (LAmB) for the treatment of candidaemia and invasive candidiasis (IC). Mycoses. 2013;56(5):532-42. http:// dx.doi.org/10.1111/myc.12071. PMid:23496163.

111. Turner SJ, Senol E, Kara A, AlBadriyeh D, Kong DC, Dinleyici EC. Pharmacoeconomic evaluation of caspofungin versus liposomal amphotericin B in empirical treatment of invasive fungal infections in Turkey. Int $\mathrm{J}$ Antimicrob Agents. 2013;42(3):27680. http://dx.doi.org/10.1016/j. ijantimicag.2013.04.030. PMid:23830892.

112. Wingard JR, Leather HL, Wood CA, Gerth WC, Lupinacci RJ, Berger $\mathrm{ML}$, et al. Pharmacoeconomic analysis of caspofungin versus liposomal amphotericin B as empirical antifungal therapy for neutropenic fever. Am J Health Syst Pharm. 2007;64(6):637-43. http:// dx.doi.org/10.2146/ajhp050521. PMid:17353573. 